\title{
Optimal risk and dividend control problem with fixed costs and salvage value: Variance premium principle
}

\author{
Dingjun Yao ${ }^{\mathrm{a}}$, Hailiang Yang ${ }^{\mathrm{b}, *}$, Rongming Wangc \\ ${ }^{a}$ School of Finance, Nanjing University of Finance and Economics, Nanjing 210023, PR China \\ ${ }^{b}$ Department of Statistics and Actuarial Science, The University of Hong Kong, Hong Kong, PR China \\ ${ }^{c}$ School of Finance and Statistics, East China Normal University, Shanghai 200241, PR China
}

\begin{abstract}
In this paper we study the combined optimal dividend, capital injection and reinsurance problems in a dynamic setting. The reinsurance premium is assumed to be calculated via the variance principle instead of the expected value principle. The proportional and fixed transaction costs and the salvage value at bankruptcy are included in the model. In both cases of unrestricted dividend rate and restricted dividend rate, we obtain the closed-form solutions of the value function and the optimal joint strategies, which depend on the transaction costs and the profitability in future.
\end{abstract}

Keywords: Dividend payment, Capital injection, Proportional reinsurance, Variance principle, Fixed costs, Salvage value.

\section{Introduction}

The classical optimal dividend problem for an insurance company consists in finding a dividend payment strategy that maximizes the total expected discounted dividends until the bankruptcy time. Much research on this issue has been carried out for various surplus process models. See, Asmussen and Taksar (1997), Gerber and Shiu (2006), Avram et al. (2007), Belhaj (2010), Azcue and Muler (2012). Capital injection is one possible way to help the manager to run the business. The company sometimes needs to raise new capitals from the market in order to continue the business. Some papers assume that the company can survive forever with forced capital injections. The expected cumulative discounted dividends minus the expected discounted costs of capital injections can be regarded as the company's value, the management seeks to find the join optimal dividend payment and capital injection strategies that maximize this value. There are many papers on this topic. For instance, Sethi and Taksar (2002), Avram et al. (2007), Kulenko and Schmidli (2008) and Yao et al. (2011). However, capital injection is not always profitable when the company is facing the financial difficulty. L $\phi$ kka and Zervos (2008) study the combined optimal dividend and capital injection problem by taking into account the possibility of bankruptcy. The optimal strategy happens to be either a dividend barrier strategy without capital injections, or another dividend barrier strategy with forced injections when surplus is null to prevent bankruptcy, which depends on the parameters of risk model. By adopting their technique, some extended results are obtained in other risk models. See, He and Liang (2008), Dai et al. (2010) and Yao et al. (2010).

Reinsurance is an effective tool for insurance companies to control the risk exposure. Due to its practical importance and theoretical value, some researchers begin to pay attention to the combined dividend and reinsurance problem. Some literature on this issue includes Asmussen and Taksar (1997), Choulli et al. (2003), Hogaard and Taksar (2004), Cadenillas et al. (2006), Meng and Siu (2011) and Peng et al. (2012). As we can see, in these literature, the expected value principle is commonly used as the reinsurance premium principle due to its simplicity and popularity in practice. Although the variance principle is another important premium principle, very few papers consider using it for risk control in a dynamic setting. Zhou and Yuen (2012) first study the optimal dividend and capital injection problem with reinsurance under the variance premium principle. Depending on whether there exist restrictions on dividend rates, they provide the optimal joint strategies in two different cases, the proportional costs for capital injections are also considered. In this paper, we continue studying the optimal dividend, capital injection and reinsurance problem with variance premium principle in a

\footnotetext{
${ }^{*}$ Corresponding author. Tel: +852 28578322; fax: +852 28589041 .

Email addresses: yaodingjun@com.cn (Dingjun Yao), hlyang@hku.hk (Hailiang Yang), rmwang@stat.ecnu.edu.cn (Rongming Wang)
} 
dynamic setting. Comparing with the work Zhou and Yuen (2012), we add the fixed costs for capital injections and a salvage value at the time of bankruptcy in our model. In real financial market, transaction cost is an unavoidable issue, especially, the fixed cost (for example, advisory and consulting fees) can generate some difficult impulse control problems. See, for example, Paulsen (2008), Bai et al. (2010), Meng and Siu (2011) and Yao et al. (2011). The salvage value of the insurer can be explained as an insurer's brand name or agency network. As we know, very little work considers optimal dividend strategies under a salvage (or penalty) for bankruptcy. A few examples are Taksar (2000), Gerber et al. (2006), Thonhauser and Albrecher (2007), Loeffen and Renaud (2010) and Liang and Young (2012). By including the fixed costs and salvage value, our model is more realistic. Under some new objective functions, we present the associated optimal joint dividend, capital injection and reinsurance strategies. We show, under our model, that the decision to declare bankruptcy or to collect new capitals depends on the model parameters, which is consistent with the results and idea in L $\phi \mathrm{kka}$ and Zervos (2008).

The outline of this paper is as follows. In Section 2, we introduce the framework of this paper and formulate two general optimization problems concerning with dividend payments, capital injections and reinsurance under the variance premium principle. In Section 3, we consider two suboptimal problems in the cases of unrestricted and restricted rates of dividend payments with forced capital injections. In Section 4, a similar study is carried out for two suboptimal problems without considering capital injections. Finally, by comparing the solutions of suboptimal problems, we identify the closed-form solutions to the general optimal problems in Section 5, which depend on the relationships among the parameters of risk model.

\section{Model formulation and the optimal control problem}

We first introduce the framework of this paper. Let $(\Omega, \mathcal{F}, \mathrm{P})$ be a probability space with the filtration $\left\{\mathcal{F}_{t}\right\}$ satisfying the usual conditions. We first present the classical insurance risk model of an insurance company, which means the surplus of an insurance company can be modeled by

$$
U_{t}=x+c t-\sum_{n=1}^{N_{t}} Y_{n}
$$

where $x$ is the initial surplus, $c$ is the premium rate, $N_{t}$ is a Poisson process with constant intensity $\lambda$, random variables $Y_{n}$ 's are positive i.i.d. claims with finite mean $\mu_{1}$ and finite second moment $\mu_{2}^{2}$. A reinsurance contract can be represented by a measurable functional $R(\cdot)$ defined on the space composed of all positive random variables such that $0 \leq R(Y) \leq Y$. Under reinsurance $R$, a positive risk $Y$ is decomposed into two parts, namely $R(Y)$ and $Y-R(Y)$, where $R(Y)$ is retained by the insurer and $Y-R(Y)$ is ceded to the reinsurer. Suppose that reinsurance $R$ is taken for each claim. Then the total ceded risk up to time $t$ is given by $\sum_{n=1}^{N_{t}}\left(Y_{n}-R\left(Y_{n}\right)\right)$, and the aggregate reinsurance premium under the variance principle takes the form

$$
\mathrm{E}\left(\sum_{n=1}^{N_{t}}\left(Y_{n}-R\left(Y_{n}\right)\right)\right)+\theta \mathrm{D}\left(\sum_{n=1}^{N_{t}}\left(Y_{n}-R\left(Y_{n}\right)\right)\right)=\lambda\left[\left(\mu_{1}-\mathrm{E}\left(R\left(Y_{1}\right)\right)+\theta \mathrm{E}\left(\left(Y_{1}-R\left(Y_{1}\right)\right)^{2}\right] t,\right.\right.
$$

where $\mathrm{E}$ and $\mathrm{D}$ stand for expectation and variance, respectively, and $\theta>0$ is a loading associated with the variance of ceded risk. Then the premium process in the presence of reinsurance $R$ can be written as

$$
U_{t}^{R}=x+\left(c-c^{R}\right) t-\sum_{n=1}^{N_{t}} R\left(Y_{n}\right)
$$

where $c^{R}=\lambda\left[\left(\mu_{1}-\mathrm{E}(R(Y))+\theta \mathrm{E}\left((Y-R(Y))^{2}\right]\right.\right.$ represents the reinsurance premium rate associated with $R$. Here we assume that the reinsurance market is frictionless. This means that the reinsurance premium rate is equal to the premium rate $c=\lambda\left(\mu_{1}+\theta \mu_{2}^{2}\right)$ if the whole risk is ceded to the reinsurer. We approximate the model (2.1) by a pure diffusion model $\left\{X_{t}^{R}, t \geq 0\right\}$ with the same drift and volatility. Specifically, $X_{t}^{R}$ satisfies the following stochastic differential equation

$$
X_{t}^{R}=x+\int_{0}^{t} \theta \lambda\left(\mu_{2}^{2}-\mathrm{E}\left(Y_{1}-R\left(Y_{1}\right)\right)^{2}\right) \mathrm{d} s+\int_{0}^{t} \sqrt{\lambda \mathrm{E}\left(\left(R\left(Y_{1}\right)\right)^{2}\right)} \mathrm{d} B_{s},
$$

with $X_{0}^{R}=x$, where $\left\{B_{t}, t \geq 0\right\}$ is a standard Brownian motion, adapted to the filtration $\mathcal{F}_{t}^{B}:=\sigma\left\{B_{s} ; 0 \leq s \leq t\right\}$. From now on, $R$ is assumed to be a proportional reinsurance policy with $R(y)=(1-a) y$. Then we represent 
$(2.2)$ as

$$
X_{t}^{a}=x+\int_{0}^{t}\left(1-a^{2}\right) \theta \lambda \mu_{2}^{2} \mathrm{~d} s+\int_{0}^{t}(1-a) \sqrt{\lambda} \mu_{2} \mathrm{~d} B_{s}
$$

with $X_{0}^{a}=x$.

Suppose that the proportion $a$ can be adjusted dynamically to control the risk exposure. Denote $L_{t}$ as the cumulative amount of dividends paid from time 0 to time $t$. The capital injection process $\left\{G_{t}=\sum_{n=1}^{\infty} I_{\left\{\tau_{n} \leq t\right\}} \eta_{n}\right\}$ is described by a sequence of increasing stopping times $\left\{\tau_{n}, n=1,2, \cdots\right\}$ and a sequence of random variables $\left\{\eta_{n}, n=1,2, \cdots\right\}$, which represent the times and the sizes of capital injections, respectively. A control strategy $\pi$ is described by $\pi=\left(a^{\pi} ; L^{\pi} ; G^{\pi}\right)=\left(a^{\pi} ; L^{\pi} ; \tau_{1}^{\pi}, \cdots \tau_{n}^{\pi}, \cdots ; \eta_{1}^{\pi}, \cdots, \eta_{n}^{\pi}, \cdots\right)$. The controlled surplus process associated with $\pi$ is given by

$$
X_{t}^{\pi}=x+\int_{0}^{t}\left(1-\left(a_{s}^{\pi}\right)^{2}\right) \theta \lambda \mu_{2}^{2} \mathrm{~d} s+\int_{0}^{t}\left(1-a_{s}^{\pi}\right) \sqrt{\lambda} \mu_{2} \mathrm{~d} B_{s}-L_{t}^{\pi}+\sum_{n=1}^{\infty} I_{\left\{\tau_{n}^{\pi} \leq t\right\}} \eta_{n}^{\pi} .
$$

Definition 2.1. A strategy $\pi=\left(a^{\pi} ; L^{\pi} ; G^{\pi}\right)$ is said to be admissible if

(i) The ceded proportion $a^{\pi}=a_{t}^{\pi}$ is an $\mathcal{F}_{t}^{B}$-adapted process with $0 \leq a_{t}^{\pi} \leq 1$ for all $t \geq 0$.

(ii) $\left\{L_{t}^{\pi}\right\}$ is an increasing, $\mathcal{F}_{t}^{B}$-adapted càdlàg process with $L_{0-}^{\pi}=0$, and satisfies that $\Delta L_{t}^{\pi}=L_{t}^{\pi}-L_{t-}^{\pi} \leq X_{t-}^{\pi}$ for all $t \geq 0$.

(iii) $\left\{\tau_{n}^{\pi}\right\}$ is a sequence of stopping times w.r.t. $\mathcal{F}_{t}^{B}$, and $0 \leq \tau_{1}^{\pi}<\cdots<\tau_{n}^{\pi}<\cdots$, a.s..

(iv) $\eta_{n}^{\pi} \geq 0, n=1,2, \cdots$ is measurable w.r.t. $\mathcal{F}_{\tau_{n}^{\pi}}^{B}$.

(v) $\mathrm{P}\left(\lim _{n \rightarrow \infty} \tau_{n}^{\pi}<T\right)=0, \forall T>0$.

The class of admissible strategies is denoted by $\Pi$.

For each strategy $\pi \in \Pi$, the bankruptcy time of the controlled process $X_{t}^{\pi}$ is defined as $\tau^{\pi}=\inf \left\{t: X_{t}^{\pi}<0\right\}$, which is an $\mathcal{F}_{t}^{B}$-stopping time. Note that the bankruptcy time could be infinite.

Problem 2.1. We define the company's value by the performance function $V(x, \pi)$, which is the expected sum of discounted salvage and the discounted dividends less the expected discounted costs of capital injections until bankruptcy

$$
V(x, \pi)=\mathrm{E}^{x}\left(\beta_{1} \int_{0}^{\tau^{\pi}} e^{-\delta s} \mathrm{~d} L_{s}^{\pi}-\sum_{n=1}^{\infty} e^{-\delta \tau_{n}^{\pi}}\left(\beta_{2} \eta_{n}^{\pi}+K\right) I_{\left\{\tau_{n}^{\pi} \leq \tau^{\pi}\right\}}+P e^{-\delta \tau^{\pi}}\right) .
$$

$\mathrm{E}^{x}$ denotes the expectation conditional on $X_{0}^{\pi}=x$, and $\delta>0$ is the discount rate. We regard $P \geq 0$ as the salvage value of the insurer; for example, an insurer's brand name or agency network which might be of value to a potential buyer of the insurer. We assume that the shareholders need to pay $\beta_{2} \eta+K$ to meet the capital injection of $\eta . \beta_{2}>1$ measures the proportional costs, $K>0$ is the fixed costs. Proportional costs on dividends transaction are taken into account through the value of $\beta_{1}$, with $0<\beta_{1} \leq 1$ representing the net proportion of leakages from the surplus received by shareholders after transaction costs have been paid. We are interested in finding the value function

$$
V(x)=\max _{\pi \in \Pi} V(x, \pi)
$$

and the associated optimal strategy $\pi^{*}$ such that $V(x)=V\left(x, \pi^{*}\right)$.

Remark 2.1. The case of $P<0$ is out of consideration in this paper. Since the surplus can keep nonnegative by ceding the whole risk to the reinsurer, so $V(0) \geq 0$ follows from the optimality of $V(x)$ and the case of $V(0)=P<0$ is impossible .

Furthermore, if we suppose that the dividend rate $l_{t}$ at time $t$ is bounded by some dividend ceiling $M>0$, then the cumulated dividend process $\left\{L_{t}, t \geq 0\right\}$ satisfies $L_{t}=\int_{0}^{t} l_{s} \mathrm{~d} s$ with $0 \leq l_{s} \leq M$.

Definition 2.2. A strategy $\bar{\pi}=\left(a^{\bar{\pi}} ; L^{\bar{\pi}} ; G^{\bar{\pi}}\right)$ is said to be admissible if

(i) The ceded proportion $a^{\bar{\pi}}=a_{t}^{\bar{\pi}}$ is an $\mathcal{F}_{t}^{B}$-adapted process with $0 \leq a_{t}^{\bar{\pi}} \leq 1$ for all $t \geq 0$. 
(ii) $\left\{L_{t}^{\bar{\pi}}, t \geq 0\right\}$ is an increasing, $\mathcal{F}_{t}^{B}$-adapted càdlàg process satisfying that $L_{0-}^{\bar{\pi}}=0$ and $L_{t}^{\bar{\pi}}=\int_{0}^{t} l_{s}^{\bar{\pi}} \mathrm{d} s$ with $0 \leq l_{s}^{\bar{\pi}} \leq M$.

(iiii) $\left\{\tau_{n}^{\bar{\pi}}\right\}$ is a sequence of stopping times w.r.t. $\mathcal{F}_{t}^{B}$, and $0 \leq \tau_{1}^{\bar{\pi}}<\cdots<\tau_{n}^{\bar{\pi}}<\cdots$, a.s..

(iv) $\eta_{n}^{\bar{\pi}} \geq 0, n=1,2, \cdots$ is measurable w.r.t. $\mathcal{F}_{\tau_{n}^{\bar{\pi}}}^{B}$.

(v) $\mathrm{P}\left(\lim _{n \rightarrow \infty} \tau_{n}^{\bar{\pi}}<T\right)=0, \forall T>0$.

The class of admissible strategies is denoted by $\bar{\Pi}$.

For each strategy $\bar{\pi} \in \bar{\Pi}$, the bankruptcy time of the controlled process $X_{t}^{\bar{\pi}}$ is defined as $\tau^{\bar{\pi}}=\inf \left\{t: X_{t}^{\bar{\pi}}<0\right\}$, which is an $\mathcal{F}_{t}^{B}$-stopping time.

Problem 2.2. Parallel to Problem 2.1, when a ceiling $M>0$ is imposed on the dividend rate, we define the following performance function

$$
V(x, \bar{\pi})=\mathrm{E}^{x}\left(\beta_{1} \int_{0}^{\tau^{\bar{\pi}}} e^{-\delta s} l_{s}^{\bar{\pi}} \mathrm{d} s-\sum_{n=1}^{\infty} e^{-\delta \tau_{n}^{\overline{\bar{\pi}}}}\left(\beta_{2} \eta_{n}^{\bar{\pi}}+K\right) I_{\left\{\tau_{n}^{\bar{\pi}} \leq \tau^{\bar{\pi}}\right\}}+P e^{-\delta \tau^{\bar{\pi}}}\right) .
$$

Correspondingly, we want to find the value function

$$
\bar{V}(x)=\max _{\bar{\pi} \in \bar{\Pi}} V(x, \bar{\pi}),
$$

and the associated optimal strategy $\bar{\pi}^{*} \in \bar{\Pi}$ such that $\bar{V}(x)=V\left(x, \bar{\pi}^{*}\right)$. Apparently, the function $\bar{V}(x)$ is bounded above by $\beta_{1} M / \delta+P$.

To develop our result, for any function $\omega(x) \in \mathbb{C}^{2}$, we define the capital injection operator $\mathscr{M}$ by

$$
\mathscr{M} \omega(x)=\max _{y \geq 0}\left\{\omega(x+y)-\beta_{2} y-K\right\}
$$

and the operator $\mathscr{A}^{a}$ with $0 \leq a \leq 1$ by

$$
\mathscr{A}^{a} \omega(x)=\frac{1}{2}(1-a)^{2} \lambda \mu_{2}^{2} \omega^{\prime \prime}(x)+\left(1-a^{2}\right) \theta \lambda \mu_{2}^{2} \omega^{\prime}(x)-\delta \omega(x) .
$$

For future use, we cite the following lemma from Zhou and Yuen (2012), which can be proved by straightforward calculations.

Lemma 2.1. Let $a(x)=(\xi+a(0)) e^{-2 \theta x}-\xi, x \geq 0$ such that $0 \leq a(0) \leq 1$. Suppose that $H(x)$ satisfies

$$
H^{\prime}(x)=\exp \left(\int_{x}^{y} \frac{2 \theta a(z)}{1-a(z)} \mathrm{d} z\right)
$$

with $(1-a(0)) H^{\prime}(0)=2 \theta \xi H(0)$ for some $\xi$ and $y>x \geq 0$. Then, we have

$$
H(x)=\frac{A(y)}{2 \theta \xi}\left(\frac{\xi+1}{\xi+a(0)} e^{2 \theta x}-1\right)^{\frac{\xi}{\xi+1}}
$$

where

$$
A(y)=(\xi+a(y))^{\frac{\xi}{\xi+1}}(1-a(y))^{\frac{\xi}{\xi+1}} .
$$

\section{The solution to the problem that does not allow for bankruptcy}

In this section we require that the company survives forever by forced capital injections. This optimization problem is studied in two cases depending on whether there exist restrictions on dividend rates. 


\subsection{Unrestricted dividends}

In this subsection, we derive closed-form solutions for the value function and the optimal strategy in the case that no restriction is imposed on the dividend rate. Denote $\pi_{r}=\left(a^{\pi_{r}} ; L^{\pi_{r}} ; G^{\pi_{r}}\right) \in \Pi$ as the controlled process such that the company never goes bankrupt. For each admissible strategy $\pi_{r}$, the performance function becomes

$$
V\left(x, \pi_{r}\right)=\mathrm{E}^{x}\left(\beta_{1} \int_{0}^{\infty} e^{-\delta s} \mathrm{~d} L_{s}^{\pi_{r}}-\sum_{n=1}^{\infty} e^{-\delta \tau_{n}^{\pi_{r}}}\left(\beta_{2} \eta_{n}^{\pi_{r}}+K\right) I_{\left\{\tau_{n}^{\pi_{r}}<\infty\right\}}\right) .
$$

The objective is to find the value function

$$
V_{r}(x)=\max _{\pi_{r} \in \Pi} V\left(x, \pi_{r}\right),
$$

and the associated optimal strategy $\pi_{r}^{*}=\left(a^{\pi_{r}^{*}} ; L^{\pi_{r}^{*}} ; G^{\pi_{r}^{*}}\right) \in \Pi$ such that $V_{r}(x)=V\left(x, \pi_{r}^{*}\right)$.

We assume that all value functions appearing in this paper are sufficiently smooth and regular. With reference to the theory of optimal control, $V_{r}(x)$ should satisfy the HJB equation and the boundary condition as follows

$$
\begin{aligned}
& \max \left\{\max _{0 \leq a \leq 1}\left\{\mathscr{A}^{a} V_{r}(x)\right\}, \beta_{1}-V_{r}^{\prime}(x), \mathscr{M} V_{r}(x)-V_{r}(x)\right\}=0, \\
& \max \left\{\mathscr{M} V_{r}(0)-V_{r}(0),-V_{r}(0)\right\}=0 .
\end{aligned}
$$

Then, we suppose the function $V_{r}$ is concave. Because the time value of money, we conjecture that the optimal timing of capital injection should only come at the moments when the surplus process hits the barrier 0 , mathematically, the equation $\mathscr{M} V_{r}(x)=V_{r}(x)$ has at most one solution at $x=0$, the inequality $\mathscr{M} V_{r}(x)<V_{r}(x)$ holds strictly for $x>0$. Actually, when the surplus reaches 0 , we have two ways to avoid bankruptcy: One way is to inject new capitals, the surplus jumps to some appropriate level $\eta^{*}>0$ immediately, if this choice is optimal, then corresponding boundary condition is $\mathscr{M} V_{r}(0)=V_{r}(0)=V_{r}\left(\eta^{*}\right)-\beta_{2} \eta^{*}-K$ and $V_{r}(0) \geq 0$. By the definition of operator $\mathscr{M}$, it follows that $\eta^{*}=\inf \left\{x: V_{r}^{\prime}(x)=\beta_{2}\right\}$. We can construct an injection strategy $G^{\pi_{r}^{*}}$ by letting

$$
\begin{aligned}
\tau_{1}^{\pi_{r}^{*}} & =\inf \left\{t \geq 0: X_{t-}^{\pi_{r}^{*}}=0\right\}, \\
\tau_{n}^{\pi_{r}^{*}} & =\inf \left\{t>\tau_{n-1}^{\pi_{r}^{*}}: X_{t-}^{\pi_{r}^{*}}=0\right\}, \quad n=2,3, \cdots, \\
\eta_{n}^{\pi_{r}^{*}} & \equiv \eta^{*}, \quad n=1,2,3, \cdots .
\end{aligned}
$$

The other way of preventing bankruptcy is to cede the whole risk to the reinsurer and keep the surplus stay at the point 0 forever, the capital injection never occurs. If this choice is optimal, the corresponding boundary condition is $V_{r}(0)=0$ and $\mathscr{M} V_{r}(0)<V_{r}(0)$. Correspondingly,

$$
G^{\pi_{r}^{*}} \equiv 0 .
$$

In addition, if we further assume that $V_{r}(x)$ is concave and there exists some number $b_{r}^{*}=\inf \left\{x: V_{r}^{\prime}(x)=\right.$ $\left.\beta_{1}\right\} \geq 0$, then the optimal dividend strategy should be a barrier strategy with the barrier $b_{r}^{*}$. Mathematically, $L_{t}^{\pi_{r}^{*}}$ satisfies

$$
L_{t}^{\pi_{r}^{*}}=\left(x-b_{r}^{*}\right)^{+}+\int_{0}^{t} I_{\left\{X_{s}^{\pi_{r}^{*}}=b_{r}^{*}\right\}} \mathrm{d} L_{s}^{\pi_{r}^{*}}
$$

In the region $\left(0, b_{r}^{*}\right)$, the optimal ceded proportion $a^{\pi_{r}^{*}}$ should satisfy

$$
\max _{0 \leq a \leq 1}\left\{\mathscr{A}^{a} V_{r}(x)\right\}=\mathscr{A}^{a^{\pi_{r}^{*}}} V_{r}(x)=0 .
$$

The optimality of strategy $\pi_{r}^{*}=\left(a^{\pi_{r}^{*}} ; L^{\pi_{r}^{*}} ; G^{\pi_{r}^{*}}\right)$ will be confirmed later.

Theorem 3.1. Let $g(x)$ be a twice continuously differentiable, increasing and concave solution of equations (3.3) and (3.4), then we have the following statements: 
(i) For each $\pi_{r} \in \Pi$, it follows that $g(x) \geq V\left(x, \pi_{r}\right)$. So $g(x) \geq V_{r}(x)$ for all $x \geq 0$.

(ii) If there exists some strategy $\pi_{r}^{*}=\left(a^{\pi_{r}^{*}} ; L^{\pi_{r}^{*}} ; G^{\pi_{r}^{*}}\right)$ such that $g(x)=V\left(x, \pi_{r}^{*}\right)$, then $g(x)=V_{r}(x)$ and $\pi_{r}^{*}$ is optimal.

Proof. The proof of $(i)$ is similar to Appendix $\mathbf{A}$, it is omitted here. The result of $(i i)$ automatically holds due to the optimality of $V_{r}(x)$.

Next, we present the following lemma, which plays a key role in finding the value function and associated optimal strategies.

Lemma 3.1. Let $\hat{a}_{0} \in[0,1]$ be the unique solution of the following equation,

$$
\beta_{1} \xi^{\frac{\xi}{\xi+1}} \frac{1}{\xi+\hat{a}_{0}}\left(\frac{\xi+1}{\xi+\hat{a}_{0}}-1\right)^{-\frac{1}{\xi+1}}=\beta_{2},
$$

where $\xi$ is a positive number. For each $a_{0} \in\left[\hat{a}_{0}, 1\right]$ and $b_{r}=\frac{1}{2 \theta} \ln \left(1+\frac{a_{0}}{\xi}\right)$, define a function

$$
\Phi_{a_{0}}(x):=\beta_{1} \xi^{\frac{\xi}{\xi+1}} \frac{e^{2 \theta x}}{\xi+a_{0}}\left(\frac{\xi+1}{\xi+a_{0}} e^{2 \theta x}-1\right)^{-\frac{1}{\xi+1}}>0, \quad 0<x \leq b_{r} .
$$

Then there is a unique $\eta \in\left(0, b_{r}\right)$ such that $\Phi_{a_{0}}(\eta)=\beta_{2}$. Furthermore,

$$
I\left(a_{0}\right):=\int_{0}^{\eta\left(a_{0}\right)}\left(\Phi_{a_{0}}(x)-\beta_{2}\right) \mathrm{d} x \geq 0, a_{0} \in\left[\hat{a}_{0}, 1\right]
$$

is an increasing function in $a_{0}$ with the range $[0, I(1)]$.

Proof. Observing that the function $\Phi_{a_{0}}(x)$ has some useful properties:

(i) The function $\Phi_{a_{0}}(x)$ is decreasing in $x$ since

$$
\Phi_{a_{0}}^{\prime}(x)=2 \theta \beta_{1} \xi^{\frac{1}{\xi+1}} \frac{e^{2 \theta x}}{\xi+a_{0}}\left(\frac{\xi+1}{\xi+a_{0}} e^{2 \theta x}-1\right)^{-\frac{1}{\xi+1}} \frac{\xi e^{2 \theta x}-\left(\xi+a_{0}\right)}{(\xi+1) e^{2 \theta x}-\left(\xi+a_{0}\right)}<0 .
$$

(ii) $\Phi_{a_{0}}\left(b_{r}\right)=\beta_{1}$ and

$$
\Phi_{a_{0}}(0)=\beta_{1} \xi^{\frac{\xi}{\xi+1}} \frac{1}{\xi+a_{0}}\left(\frac{\xi+1}{\xi+a_{0}}-1\right)^{-\frac{1}{\xi+1}} .
$$

(iii) For each fixed $0 \leq x \leq b_{r}, \Phi_{a_{0}}(x)$ can be viewed as an increasing function of $a_{0}$. Especially,

$$
\lim _{a_{0} \rightarrow 1-} \Phi_{a_{0}}(0)=+\infty .
$$

(iv) Obviously, (3.11) can be written as $\Phi_{\hat{a}_{0}}(0)=\beta_{2}$.

Based on above analysis, we conclude that there exists a unique solution $\eta\left(a_{0}\right) \in\left[0, b_{r}\right)$ to equation $\Phi_{a_{0}}(\eta)=$ $\beta_{2}$ if $a_{0} \in\left[\hat{a}_{0}, 1\right]$. More importantly, $\eta\left(a_{0}\right)$ is an increasing function of $a_{0}$. The minimum $\eta_{\text {min }}=\eta\left(\hat{a}_{0}\right)=0$ and the maximum $\eta_{\max }=\eta(1)<b_{r}$ is uniquely determined by

$$
\Phi_{1}(\eta(1))=\beta_{1} \xi^{\frac{\xi}{\xi+1}} \frac{e^{2 \theta \eta(1)}}{\xi+1}\left(e^{2 \theta \eta(1)}-1\right)^{-\frac{1}{\xi+1}}=\beta_{2} .
$$

Thereby, together with $(i i i), I\left(a_{0}\right)$ is non-negative and increasing on $\left(\hat{a}_{0}, 1\right]$. It is easy to verify that

$$
I\left(\hat{a}_{0}\right)=0 \quad \text { and } \quad I(1)=\int_{0}^{\eta(1)}\left(\Phi_{1}(x)-\beta_{2}\right) \mathrm{d} x \leq \frac{\beta_{1}}{2 \theta \xi} .
$$

Thereby, $I\left(a_{0}\right) \in[0, I(1)]$ for $a_{0} \in\left[\hat{a}_{0}, 1\right]$.

Theorem 3.2. The value function $V_{r}(x)$ coincides with

$$
g(x)= \begin{cases}\beta_{1}\left(x-b_{r}^{*}\right)+\frac{\beta_{1}}{2 \theta \xi}, & x \geq b_{r}^{*}, \\ \frac{\beta_{1}}{2 \theta} \xi^{-\frac{1}{\xi+1}}\left(\frac{\xi+1}{\xi+a^{\pi *}(0)} e^{2 \theta x}-1\right)^{\frac{\xi}{\xi+1}}, & 0 \leq x \leq b_{r}^{*},\end{cases}
$$


where $b_{r}^{*}=\frac{1}{2 \theta} \ln \left(1+\frac{a^{\pi_{r}^{*}}(0)}{\xi}\right)$. Correspondingly, the optimal dividend strategy $L^{\pi_{r}^{*}}$ satisfies

$$
L_{t}^{\pi_{r}^{*}}=\left(x-b_{r}^{*}\right)^{+}+\int_{0}^{t} I_{\left\{X_{s}^{\pi_{r}^{*}}=b_{r}^{*}\right\}} \mathrm{d} L_{s}^{\pi_{r}^{*}} .
$$

The optimal ceded proportion $a^{\pi_{r}^{*}}$ coincides with

$$
a^{\pi_{r}^{*}}(x)= \begin{cases}0, & x>b_{r}^{*}, \\ \left(\xi+a^{\pi_{r}^{*}}(0)\right) e^{-2 \theta x}-\xi, & 0 \leq x \leq b_{r}^{*},\end{cases}
$$

which solves (3.10). The value of $a^{\pi_{r}^{*}}(0)$ and the injection strategy $G^{\pi_{r}^{*}}$ can be determined as follows:

( $i$ ) In the case of $0<K \leq I(1), a^{\pi_{r}^{*}}(0)=a_{0} \in\left(\hat{a}_{0}, 1\right]$ is the unique solution of $I\left(a_{0}\right)=K$. The optimal injection strategy $G^{\pi_{r}^{*}}$ is described by (3.5)-(3.7), the value of $\eta^{*}$ can be obtained by (3.32) and (3.33). It means the surplus immediately jumps to $\eta^{*}$ once it reaches 0 by injecting capitals. In this case, it follows that $\mathscr{M} g(0)=g(0)$ and $g(0) \geq 0$.

(ii) In the case of $K \geq I(1)$, the initial value is $a^{\pi_{r}^{*}}(0)=1$, and the injection strategy $G_{t}^{\pi_{r}^{*}}$ in (3.8) is optimal. Namely, the capital injection never occurs. When the surplus reaches 0 , the company cedes the whole business to the reinsurer and keeps the surplus stay at the point 0 forever. In this case, $\mathscr{M} g(0) \leq g(0)$ and $g(0)=0$.

Proof. We try to find a concave solution of (3.3) and (3.4) with a switch point $b_{r}^{*}$ such that $g^{\prime}\left(b_{r}^{*}\right)=\beta_{1}$. We conjecture that $g^{\prime}(x) \equiv \beta_{1}$ holds for all $x \geq b_{r}^{*}$, which yields

$$
g(x)=\beta_{1}\left(x-b_{r}^{*}\right)+g\left(b_{r}^{*}\right) .
$$

Moreover the concavity implies that $g^{\prime}(x) \geq \beta_{1}$ for $x \leq b_{r}^{*}$, to satisfy (3.3), it must have

$$
\max _{0 \leq a \leq 1}\left\{\mathscr{A}^{a} g(x)\right\}=0, \quad 0 \leq x \leq b_{r}^{*}
$$

specifically,

$$
\max _{0 \leq a \leq 1}\left\{\frac{1}{2}(1-a)^{2} \lambda \mu_{2}^{2} g^{\prime \prime}(x)+\left(1-a^{2}\right) \theta \lambda \mu_{2}^{2} g^{\prime}(x)-\delta g(x)\right\}=0 .
$$

Taking derivative with respect to $a$ and setting the derivative equal to zero yield

$$
\frac{g^{\prime \prime}(x)}{g^{\prime}(x)}=-2 \theta \frac{a^{\pi_{r}^{*}}(x)}{1-a^{\pi_{r}^{*}}(x)},
$$

where $a^{\pi_{r}^{*}}(x)$ is the maximizer in (3.24). Plugging (3.25) in (3.24) yields

$$
\left(1-a^{\pi_{r}^{*}}(x)\right) g^{\prime}(x)=2 \theta \xi g(x),
$$

with $\xi=\delta /\left(2 \lambda\left(\theta \mu_{2}\right)^{2}\right)>0$. Taking derivative with respect to $x$ on both sides of (3.26) and using (3.25), we obtain

$$
a^{\pi_{r}^{*}}(x)=\left(\xi+a^{\pi_{r}^{*}}(0)\right) e^{-2 \theta x}-\xi
$$

Note that we have $a^{\pi_{r}^{*}}\left(x_{0}\right)=0$ with

$$
x_{0}=\frac{1}{2 \theta} \ln \left(1+\frac{a^{\pi_{r}^{*}}(0)}{\xi}\right) .
$$

For $0 \leq x \leq x_{0}$, it follows that from (3.26) that

$$
g^{\prime}(x)=k \exp \left(\int_{x}^{x_{0}} \frac{2 \theta a^{\pi_{r}^{*}}(z)}{1-a^{\pi_{r}^{*}}(z)} \mathrm{d} z\right)
$$

with $g^{\prime}\left(x_{0}\right)=k$ and

$$
g^{\prime \prime}\left(x_{0}\right)=-2 \theta k \frac{a^{\pi_{r}^{*}}\left(x_{0}\right)}{1-a^{\pi_{r}^{*}}\left(x_{0}\right)} g^{\prime}\left(x_{0}\right)=0
$$


the constant $k$ needs to be determined. Applying Lemma 2.1 to (3.26) and (3.29) yields

$$
g(x)=\frac{1}{2 \theta} k \xi^{-\frac{1}{\xi+1}}\left(\frac{\xi+1}{\xi+a^{\pi_{r}^{*}}(0)} e^{2 \theta x}-1\right)^{\frac{\xi}{1+\xi}}, \quad 0 \leq x \leq x_{0},
$$

with $g\left(x_{0}\right)=k /(2 \theta \xi)$. We conjecture that there is only one common switch level for the optimal reinsurance and dividend strategies, i.e., $x_{0}=b_{r}^{*}$. Then it follows that $k=g^{\prime}\left(x_{0}\right)=g^{\prime}\left(b_{r}^{*}\right)=\beta_{1}$. By the way, we can deduce that $g\left(b_{r}^{*}\right)=\frac{\beta_{1}}{2 \theta \xi}$. The unknown value of $a^{\pi_{r}^{*}}(0)$ and the optimal injection process $G_{r}^{\pi_{r}^{*}}$ can be determined in different cases:

(i) In the case of $0<K \leq I(1)$, we conjecture that $\mathscr{M} g(0)=g(0)$ holds. It requires that there exist some $a^{\pi_{r}^{*}}(0) \in[0,1]$ and $\eta^{*}\left(a^{\pi_{r}^{*}}(0)\right)>0$ such that

$$
\begin{aligned}
& g^{\prime}\left(\eta^{*}\right)=\beta_{2}, \\
& g(0)=g\left(\eta^{*}\right)-\beta_{2} \eta^{*}-K=\mathscr{M} g(0) .
\end{aligned}
$$

(3.33) can be rewritten as

$$
K=\int_{0}^{\eta^{*}}\left(g^{\prime}(x)-\beta_{2}\right) \mathrm{d} x=\int_{0}^{\eta^{*}\left(a_{r}^{\pi_{r}^{*}}(0)\right)}\left(\Phi_{a^{\pi_{r}^{*}}(0)}(x)-\beta_{2}\right) \mathrm{d} x=I\left(a^{\pi_{r}^{*}}(0)\right) .
$$

Recalling Lemma 3.1, we know that $a^{\pi_{r}^{*}}(0) \in\left(\hat{a}_{0}, 1\right]$ and $\eta^{*}$ exist if $0<K \leq I(1)$.

A straightforward calculation can verify that $g(x)$ is indeed a twice continuously differentiable, increasing and concave function. Now, according to Theorem 3.1, we are in the position to verify that $g(x)$ in $(3.19)$ satisfies (3.3) and (3.4). From the construction of $g(x)$, we know that $g^{\prime}(x)=\beta_{1}$ for $x \geq b_{r}^{*}$ and $\max _{0 \leq a \leq 1}\left\{\mathscr{A}^{a} g(x)\right\}=0$ for $0 \leq x \leq b_{r}^{*}$. In addition, from the expression of $g(x)$ for $x \geq b_{r}^{*}$ we have

$$
\begin{aligned}
\max _{0 \leq a \leq 1}\left\{\mathscr{A}^{a} g(x)\right\} & =\max _{0 \leq a \leq 1}\left\{\left(1-a^{2}\right) \theta \lambda \mu_{2}^{2} \beta_{1}-\delta\left[\beta_{1}\left(x-b_{r}^{*}\right)+\frac{\beta_{1}}{2 \theta \xi}\right]\right\} \\
& =\theta \lambda \mu_{2}^{2} \beta_{1}-\delta\left[\beta_{1}\left(x-b_{r}^{*}\right)+\frac{\beta_{1}}{2 \theta \xi}\right] \\
& =-\delta \beta_{1}\left(x-b_{r}^{*}\right) \leq 0
\end{aligned}
$$

and, for $0 \leq x \leq b_{r}^{*}$, the property $g^{\prime}(x) \leq \beta_{1}$ follows from the conditions $g^{\prime}\left(b_{r}^{*}\right)=\beta_{1}$ and $g^{\prime \prime}(x) \leq 0$. Moreover, $g(0)=\mathscr{M} g(0)$ and $g(0) \geq 0$ are obvious. Hence, $g(x)$ in (3.19) satisfies HJB equation (3.3) and (3.4). Finally, the optimality of $\pi_{r}^{*}$ can be verified by Appendix B. According to Theorem 3.1, the statements are proved.

(ii) In the case of $K \geq I(1)$, (3.19)-(3.21) can also be obtained. However, the number $\eta^{*}$ satisfying (3.32) and (3.33) does not exist and $\mathscr{M} g(0)<g(0)$, which suggests that $G_{r}^{\pi_{r}^{*}} \equiv 0$. To satisfy (3.4), it must be true that $g(0)=0$ and $a^{\pi_{r}^{*}}(0)=1$. By repeating the same verification process as that in $(i)$, we can prove that $g(x)=V_{r}(x)=V\left(x, \pi_{r}^{*}\right)$.

Remark 3.1. Theorem 3.2 suggests that the company should give up to raise new capitals from market if the fixed cost $K$ is larger than $I(1)$. It can be viewed as the maximal fixed cost for injections that the company would pay. Observe from (3.12) and (3.18), I(1) becomes larger when the cost factor for injections $\beta_{2}$ decreases or the cost factor for dividends $\beta_{1}$ increases. These results agree with our intuition.

\subsection{Restricted dividends}

In this subsection, we derive explicit solutions for the value function and the optimal strategy when a ceiling $M>0$ is imposed on the dividend rate. Denote $\bar{\pi}_{r} \in \bar{\Pi}$ as the control strategy such that the company never goes bankrupt. The performance function associated with $\bar{\pi}_{r}$ takes the form of

$$
V\left(x, \bar{\pi}_{r}\right)=\mathrm{E}^{x}\left(\beta_{1} \int_{0}^{\infty} e^{-\delta s} l_{s}^{\bar{\pi}_{r}} \mathrm{~d} s-\sum_{n=1}^{\infty} e^{-\delta \tau_{n}^{\bar{\pi}_{r}}}\left(\beta_{2} \eta_{n}^{\bar{\pi}_{r}}+K\right) I_{\left\{\tau_{n}^{\bar{\pi}_{r}}<\infty\right\}}\right) .
$$

We are interesting in the value function as

$$
\bar{V}_{r}(x)=\max _{\bar{\pi}_{r} \in \bar{\Pi}} V\left(x, \bar{\pi}_{r}\right),
$$


and the optimal strategy $\bar{\pi}_{r}^{*}=\left(a^{\bar{\pi}_{r}^{*}} ; L^{\bar{\pi}_{r}^{*}} ; G^{\bar{\pi}_{r}^{*}}\right) \in \bar{\Pi}$, where $\bar{V}_{r}(x)=V\left(x, \bar{\pi}_{r}^{*}\right)$.

With reference to the theory of optimal control, $\bar{V}_{r}(x)$ should satisfy the HJB equation and the boundary condition as follows

$$
\begin{aligned}
& \max \left\{\max _{0 \leq \bar{a} \leq 1,0 \leq l \leq M}\left\{\mathscr{A}^{\bar{a}} \bar{V}_{r}(x)+l\left(\beta_{1}-\bar{V}_{r}^{\prime}(x)\right)\right\}, \mathscr{M} \bar{V}_{r}(x)-\bar{V}_{r}(x)\right\}=0, \\
& \max \left\{\mathscr{M} \bar{V}_{r}(0)-\bar{V}_{r}(0),-\bar{V}_{r}(0)\right\}=0 .
\end{aligned}
$$

Similar to the analysis in Subsection 3.1, we assume that $\bar{V}_{r}(x)$ is concave and the equation $\mathscr{M} \bar{V}_{r}(x)=\bar{V}_{r}(x)$ has at most one solution at $x=0$, the inequality $\mathscr{M} \bar{V}_{r}(x)<\bar{V}_{r}(x)$ holds strictly for $x>0$. When the surplus reaches 0 , we have two ways to avoid bankruptcy: One way is to inject new capitals, the surplus jumps to some appropriate level $\bar{\eta}^{*}>0$ immediately, if this choice is optimal, then the corresponding boundary condition is $\mathscr{M} \bar{V}_{r}(0)=\bar{V}_{r}(0)=\bar{V}_{r}\left(\bar{\eta}^{*}\right)-\beta_{2} \bar{\eta}^{*}-K$ and $\bar{V}_{r}(0) \geq 0$. By the definition of operator $\mathscr{M}$, it follows that $\bar{\eta}^{*}=\inf \left\{x: \bar{V}_{r}^{\prime}(x)=\beta_{2}\right\}$. We can construct an injection strategy $G_{r}^{\bar{\pi}_{r}^{*}}$ by letting

$$
\begin{aligned}
\tau_{1}^{\bar{\pi}_{r}^{*}} & =\inf \left\{t \geq 0: X_{t-}^{\bar{\pi}_{r}^{*}}=0\right\}, \\
\tau_{n}^{\bar{\pi}_{r}^{*}} & =\inf \left\{t>\tau_{n-1}^{\bar{\pi}_{r}^{*}}: X_{t-}^{\bar{\pi}_{r}^{*}}=0\right\}, \quad n=2,3, \cdots, \\
\eta_{n}^{\bar{\pi}_{r}^{*}} & \equiv \bar{\eta}^{*}, \quad n=1,2,3, \cdots .
\end{aligned}
$$

The other way of preventing bankruptcy is to cede the whole business to the reinsurer and keep the surplus stay at the point 0 forever, the capital injection never occurs. If this choice is optimal, the boundary condition becomes $\bar{V}_{r}(0)=0$ and $\mathscr{M} \bar{V}_{r}(0)<\bar{V}_{r}(0)$. Correspondingly,

$$
G_{t}^{\bar{\pi}_{r}^{*}} \equiv 0
$$

If we further assume that $\bar{V}_{r}(x)$ is concave and there exists some number $\bar{b}_{r}^{*}=\inf \left\{x: \bar{V}_{r}^{\prime}(x)=\beta_{1}\right\} \geq 0$, then the threshold dividend strategy $L_{r}^{\bar{\pi}_{r}^{*}}$ with the following dividend rate

$$
l^{\bar{\pi}_{r}^{*}}=\bar{l}_{r}^{*}(x)=\left\{\begin{array}{l}
M, \quad x>\bar{b}_{r}^{*}, \\
0, \quad 0 \leq x \leq \bar{b}_{r}^{*},
\end{array}\right.
$$

is optimal. The optimal ceded proportion strategy $a^{\bar{\pi}_{r}^{*}}$ should satisfy

$$
\max _{0 \leq \bar{a} \leq 1} \mathscr{A}^{\bar{a}} \bar{V}_{r}(x)=\mathscr{A}^{a^{\bar{\pi}_{r}^{*}}} \bar{V}_{r}(x)=0, \quad 0 \leq x \leq \bar{b}_{r}^{*} .
$$

However, in the region $\left[\bar{b}_{r}^{*}, \infty\right)$, the optimal ceded proportion is expected to be some constant $\bar{a}^{*} \in\left[0, a^{\bar{\pi}_{r}^{*}}(0)\right]$. The optimality of strategy $\bar{\pi}_{r}^{*}=\left(a^{\bar{\pi}_{r}^{*}} ; L_{r}^{\bar{\pi}_{r}^{*}} ; G^{\bar{\pi}_{r}^{*}}\right)$ will be established later.

Theorem 3.3. Let $w(x)$ be a twice continuously differentiable, increasing and concave solution to (3.38) and (3.39). Then, we have the following statements:

(i) For each $\bar{\pi}_{r} \in \bar{\Pi}$, it follows that $w(x) \geq V\left(x, \bar{\pi}_{r}\right)$. So $w(x) \geq \bar{V}_{r}(x)$ for all $x \geq 0$.

(ii) If there exists some strategy $\bar{\pi}_{r}^{*}=\left(a^{\bar{\pi}_{r}^{*}} ; L^{\bar{\pi}_{r}^{*}} ; G^{\bar{\pi}_{r}^{*}}\right)$ such that $w(x)=V\left(x, \bar{\pi}_{r}^{*}\right)$, then $w(x)=\bar{V}_{r}(x)$ and $\bar{\pi}_{r}^{*}$ is optimal.

Proof. The proof of $(i)$ is similar to Appendix A, it is omitted here. The result of $(i i)$ comes from the optimality of $\bar{V}_{r}(x)$.

Analogous to Lemma 3.1, we state the following lemma for future reference, its proof is omitted because it is quite similar to the proof of Lemma 3.1.

Lemma 3.2. Let $\tilde{a}_{0} \in[0,1]$ be the unique solution of the following equation,

$$
\frac{A}{\xi+\tilde{a}_{0}}\left(\frac{\xi+1}{\xi+\tilde{a}_{0}}-1\right)^{-\frac{1}{\xi+1}}=\beta_{2}
$$


with some $A=\beta_{1}\left(\xi+\bar{a}^{*}\right)^{\frac{\xi}{\xi+1}}\left(1-\bar{a}^{*}\right)^{\frac{1}{\xi+1}}$ and $\bar{a}^{*} \geq 0$. For any number $\bar{a}_{0} \in\left[\tilde{a}_{0}, 1\right]$ and $\bar{b}_{r}=\frac{1}{2 \theta} \ln \left(\frac{\xi+\bar{a}_{0}}{\xi+\bar{a}^{*}}\right)$, define a function

$$
\Psi_{\bar{a}_{0}}(x):=A \frac{e^{2 \theta x}}{\xi+\bar{a}_{0}}\left(\frac{\xi+1}{\xi+\bar{a}_{0}} e^{2 \theta x}-1\right)^{-\frac{1}{\xi+1}}>0, \quad 0 \leq x \leq \bar{b}_{r} .
$$

Then there is a unique $\bar{\eta} \in\left[0, \bar{b}_{r}\right)$ such that $\Psi_{\bar{a}_{0}}(\bar{\eta})=\beta_{2}$. Furthermore,

$$
\bar{I}\left(\bar{a}_{0}\right)=\int_{0}^{\bar{\eta}\left(\bar{a}_{0}\right)}\left(\Psi_{\bar{a}_{0}}(x)-\beta_{2}\right) \mathrm{d} x \geq 0, \quad \bar{a}_{0} \in\left[\tilde{a}_{0}, 1\right],
$$

is an increasing function in $\bar{a}_{0}$ with the range $[0, \bar{I}(1)]$. In addition, it is not difficult to show that

$$
\bar{I}\left(\tilde{a}_{0}\right)=0 \quad \text { and } \quad \bar{I}(1) \leq \frac{\beta_{1}\left(1-\bar{a}^{*}\right)}{2 \theta \xi} .
$$

Theorem 3.4. If the dividend rate is bounded by $0<M<\infty$, then the value function $\bar{V}_{r}(x)$ coincides with

$$
w(x)=\left\{\begin{array}{lc}
\frac{\beta_{1} M}{\delta}+\frac{\beta_{1}}{\gamma} e^{\gamma\left(x-\bar{b}_{r}^{*}\right)}, & x>\bar{b}_{r}^{*}, \\
\frac{A}{2 \theta \xi}\left(\frac{\xi+1}{\xi+a^{\pi_{r}^{*}}(0)} e^{2 \theta x}-1\right)^{\frac{\xi}{\xi+1}}, & 0 \leq x \leq \bar{b}_{r}^{*},
\end{array}\right.
$$

where $A=\beta_{1}\left(\xi+\bar{a}^{*}\right)^{\frac{\xi}{\xi+1}}\left(1-\bar{a}^{*}\right)^{\frac{1}{\xi+1}}, \bar{b}_{r}^{*}=\frac{1}{2 \theta} \ln \left(\frac{\xi+a^{\bar{\pi}_{r}^{*}}(0)}{\xi+\bar{a}^{*}}\right), \gamma<0$ and $\bar{a}^{*} \in[0,1]$ are unique roots of (3.59) and (3.60), respectively. The threshold dividend strategy $L^{\bar{\pi}_{r}^{*}}$ defined by $\bar{l}_{r}^{*}$ in (3.44) is optimal. The optimal ceded proportion $a^{\bar{\pi}_{r}^{*}}$ takes the form as

$$
a^{\bar{\pi}_{r}^{*}}(x)= \begin{cases}\bar{a}^{*}, & x>\bar{b}_{r}^{*}, \\ \left(\xi+a^{\bar{\pi}_{r}^{*}}(0)\right) e^{-2 \theta x}-\xi, & 0<x \leq \bar{b}_{r}^{*} .\end{cases}
$$

The value of $a^{\bar{\pi}_{r}^{*}}(0)$ and injection strategy $G^{\bar{\pi}_{r}^{*}}$ is given according to two different cases:

(i) In the case of $0<K \leq \bar{I}(1)$, the initial value $a^{\bar{\pi}_{r}^{*}}(0)=\bar{a}_{0} \in[0,1]$ is the unique solution of $\bar{I}\left(\bar{a}_{0}\right)=K$. The injection strategy $G^{\bar{\pi}_{r}^{*}}$ is described by (3.40)-(3.42), in which the value of $\bar{\eta}^{*}$ will be given in (3.62) and (3.63). It means the surplus immediately jumps to $\bar{\eta}^{*}$ once it reaches 0 by injecting capital. In this case, it follows that $\mathscr{M} w(0)=w(0)$ and $w(0) \geq 0$.

(ii) In the case of $K \geq \bar{I}(1)$, the ceded proportion described by (3.51) with the initial value $a^{\bar{\pi}_{r}^{*}}(0)=1$ is optimal. However, the optimal strategy of capital injection is (3.43), namely, the capital injection never occurs. Whenever the surplus reaches 0 , one lets the ceded proportion of risk be $a^{\bar{\pi}_{r}^{*}}(0)=1$ and keeps the surplus stay at the point 0 forever. In this case, $\mathscr{M} w(0) \leq w(0)$ and $w(0)=0$.

Proof. We try to find a concave solution $w(x)$ of (3.38) with a switch level $\bar{b}_{r}^{*}>0$ such that $w^{\prime}\left(\bar{b}_{r}^{*}\right)=\beta_{1}$. Then we have $w^{\prime}(x) \geq \beta_{1}$ for $0 \leq x \leq \bar{b}_{r}^{*}$, so

$$
\max _{0 \leq \bar{a} \leq 1,0 \leq l \leq M}\left\{\mathscr{A}^{\bar{a}} w(x)+l\left(\beta_{1}-w^{\prime}(x)\right)\right\}=\max _{0 \leq \bar{a} \leq 1}\left\{\mathscr{A}^{\bar{a}} w(x)\right\}=0, \quad 0 \leq x \leq \bar{b}_{r}^{*},
$$

specifically,

$$
\max _{0 \leq \bar{a} \leq 1}\left\{\frac{1}{2}(1-\bar{a})^{2} \lambda \mu_{2}^{2} w^{\prime \prime}(x)+\left(1-\bar{a}^{2}\right) \theta \lambda \mu_{2}^{2} w^{\prime}(x)-\delta w(x)\right\}=0, \quad 0 \leq x \leq \bar{b}_{r}^{*} .
$$

Following the same method as that in Theorem 3.2, we provide the candidate solution to (3.53)

$$
w(x)=\frac{A}{2 \theta \xi}\left(\frac{\xi+1}{\xi+a_{r}^{\bar{\pi}_{r}^{*}}(0)} e^{2 \theta x}-1\right)^{\frac{\xi}{1+\xi}}, \quad 0 \leq x \leq \bar{b}_{r}^{*},
$$

with optimal ceded proportion $a^{\bar{\pi}_{r}^{*}}(x)$ as

$$
a^{\bar{\pi}_{r}^{*}}(x)=\left(\xi+a^{\bar{\pi}_{r}^{*}}(0)\right) e^{-2 \theta x}-\xi, \quad 0 \leq x \leq \bar{b}_{r}^{*} .
$$


Applying the condition $w\left(\bar{b}_{r}^{*}-\right)=\beta_{1}$ to $(3.54)$ yields $A=\left(\xi+\bar{a}^{*}\right)^{\frac{\xi}{\xi+1}}\left(1-\bar{a}^{*}\right)^{\frac{1}{\xi+1}}$. For $x>\bar{b}_{r}^{*}$, it has $w^{\prime}(x)<\beta_{1}$, we conjecture the following equation holds with some constant $0 \leq \bar{a}^{*} \leq 1$,

$$
\max _{0 \leq \bar{a} \leq 1,0 \leq l \leq M}\left\{\mathscr{A}^{\bar{a}} w(x)+l\left(\beta_{1}-w^{\prime}(x)\right)\right\}=\mathscr{A}^{\bar{a}^{*}} w(x)+M\left(\beta_{1}-w^{\prime}(x)\right)=0,
$$

or equivalently,

$$
\frac{1}{2}\left(1-\bar{a}^{*}\right)^{2} \lambda \mu_{2}^{2} w^{\prime \prime}(x)+\left[\left(1-\bar{a}^{* 2}\right) \theta \lambda \mu_{2}^{2}-M\right] w^{\prime}(x)-\delta w(x)+\beta_{1} M=0 .
$$

Recalling that $w(x)$ should be bounded and $w^{\prime}\left(\bar{b}_{r}^{*}\right)=\beta_{1}$, then

$$
w(x)=\frac{\beta_{1} M}{\delta}+\frac{\beta_{1}}{\gamma} e^{\gamma\left(x-\bar{b}_{r}^{*}\right)},
$$

where $\gamma$ is the negative root of the equation

$$
\frac{1}{2}\left(1-\bar{a}^{*}\right)^{2} \lambda \mu_{2}^{2} \gamma^{2}+\left[\left(1-\bar{a}^{* 2}\right) \theta \lambda \mu_{2}^{2}-M\right] \gamma-\delta=0 .
$$

To match the continuous condition

$$
\frac{w^{\prime \prime}\left(\bar{b}_{r}^{*}-\right)}{w^{\prime}\left(\bar{b}_{r}^{*}-\right)}=\frac{w^{\prime \prime}\left(\bar{b}_{r}^{*}+\right)}{w^{\prime}\left(\bar{b}_{r}^{*}+\right)}
$$

it requires that

$$
-\frac{2 \theta \bar{a}^{*}}{1-\bar{a}^{*}}=\gamma
$$

Then, by substituting (3.59) into (3.58), we see that $0<\bar{a}^{*}<1$ is the unique positive root of following equation in $a$

$$
a^{2}+\left(\xi-1+\frac{2 M \theta \xi}{\delta}\right) a-\xi=0
$$

In addition, the continuity of $a^{\bar{\pi}_{r}^{*}}(x)$ at point $\bar{b}_{r}^{*}$ shows

$$
\left(\xi+a^{\bar{\pi}_{r}^{*}}(0)\right) e^{-2 \theta \bar{b}_{r}^{*}}-\xi=\bar{a}^{*}
$$

which yields $\bar{b}_{r}^{*}=\frac{1}{2 \theta} \ln \left(\frac{\xi+a^{\bar{\pi}_{r}^{*}}(0)}{\xi+\bar{a}^{*}}\right)$. The unknown value of $a^{\bar{\pi}_{r}^{*}}(0)$ and the optimal injection strategy $G^{\bar{\pi}_{r}^{*}}$ can be determined as follows:

(i) Relying on Lemma 3.2 and the fact that $w^{\prime}(x)=\Psi_{a^{\bar{\pi}_{r}^{*}(0)}}(x)$, when $0<K \leq \bar{I}(1)$ there exists a unique value $\bar{\eta}^{*}$ such that

$$
\begin{aligned}
& w^{\prime}\left(\bar{\eta}^{*}\right)=\beta_{2}, \\
& w(0)=w\left(\bar{\eta}^{*}\right)-\beta_{2} \bar{\eta}^{*}-K=\mathscr{M} w(0) .
\end{aligned}
$$

$a^{\bar{\pi}_{r}^{*}}(0)=\bar{a}_{0}$ is the unique solution of $\bar{I}\left(\bar{a}_{0}\right)=K$.

Finally, a straightforward calculation can verify that $w(x)$ is indeed a twice continuously differentiable, increasing and concave solution of (3.38) and (3.39), moreover, $w(x)=V\left(x, \bar{\pi}_{r}^{*}\right)$ can be proved as that in Appendix B. According to Theorem 3.3, the results are proved.

(ii) In the case of $K \geq \bar{I}(1)$, by repeating the same discussion as above, $\bar{V}_{r}(x), a^{\bar{\pi}_{r}^{*}}$ and $L^{\bar{\pi}_{r}^{*}}$ can also be given by (3.50), (3.51) and (3.44) respectively, apart from different initial value of $a^{\bar{\pi}_{r}^{*}}(0)$. In this case, $\bar{\eta}^{*}$ satisfying (3.62) and (3.63) does not exist, i.e., $\mathscr{M} w(0)<w(0)$. Thus the optimal capital injection process is $G_{t}^{\bar{\pi}_{r}^{*}} \equiv 0$. The capital injection never occurs. To satisfy (3.39), the condition $w(0)=0$ is required, which yields $a^{\bar{\pi}_{r}^{*}}(0)=1$. Whenever the surplus reaches 0 , let the ceded proportion of risk be $a^{\bar{\pi}_{r}^{*}}(0)=1$ and keep the surplus stay at the point 0 forever.

Finally, $w(x)$ is indeed a twice continuously differentiable, increasing and concave solution of (3.38) and 
(3.39). Similar to Appendix $\mathbf{B}, w(x)=\bar{V}_{r}(x)=V\left(x, \bar{\pi}_{r}^{*}\right)$ can also be proved.

Remark 3.2. Parallel to Remark 3.1, Theorem 3.4 suggests that the company should give up to raise new capitals from market if the fixed cost $K$ is larger than $\bar{I}(1)$. It can be viewed as the maximal fixed cost for injections that the company would afford. Notice, from (3.47) and (3.48), that $\bar{I}(1)$ becomes larger as the cost factor for injection $\beta_{2}$ decreases or the cost factor for dividend $\beta_{1}$ increases. These results are consistent with our intuition.

\section{The solution to the problem without capital injection}

\subsection{Unrestricted dividends}

In this subsection, we consider the classical optimal dividend problem of maximizing the expected total discounted dividends under a salvage for bankruptcy and the dividend rate is not restricted. Let $\pi_{p}=$ $\left(a^{\pi_{p}} ; L^{\pi_{p}} ; 0\right) \in \Pi$ stand for the control process in which capital injection is not allowed. Then the performance function associated with $\pi_{p}$ is defined by

$$
V\left(x, \pi_{p}\right)=\mathrm{E}^{x}\left(\beta_{1} \int_{0}^{\tau^{\pi_{p}}} e^{-\delta s} \mathrm{~d} L_{s}^{\pi_{p}}+P e^{-\delta \tau^{\pi_{p}}}\right) .
$$

Correspondingly, the value function is defined by

$$
V_{p}(x)=\max _{\pi_{p} \in \Pi} V\left(x, \pi_{p}\right) .
$$

The associated optimal strategy is $\pi_{p}^{*}=\left(a^{\pi_{p}^{*}} ; L^{\pi_{p}^{*}} ; 0\right)$ such that $V_{p}(x)=V\left(x, \pi_{p}^{*}\right)$ needs to be determined. From the optimal control theory, $V_{p}(x)$ should satisfy the following HJB equation

$$
\max \left\{\max _{0 \leq a \leq 1} \mathscr{A}^{a} V_{p}(x), \beta_{1}-V_{p}^{\prime}(x)\right\}=0
$$

with the boundary condition

$$
V_{p}(0)=P .
$$

Theorem 4.1. Let $f(x)$ be a twice continuously differentiable, increasing and concave solution of equations (4.3) and (4.4), then we have the following statements:

(i) For each $\pi_{p} \in \Pi$, we have $g(x) \geq V\left(x, \pi_{p}\right)$. So $f(x) \geq V_{p}(x)$ for all $x \geq 0$.

(ii) If there exists some strategy $\pi_{p}^{*}=\left(a^{\pi_{p}^{*}} ; L^{\pi_{p}^{*}} ; 0\right)$ such that $f(x)=V\left(x, \pi_{p}^{*}\right)$, then $f(x)=V_{p}(x)$ and $\pi_{p}^{*}$ is optimal.

Proof. The proof of $(i)$ is similar to Appendix A, it is omitted here. The result of $(i i)$ comes from the optimality of $V_{p}(x)$.

Theorem 4.2. The value function $V_{p}(x)$ and the associated optimal strategy $\pi_{p}^{*}=\left(a_{p}^{\pi_{p}^{*}} ; L^{\pi_{p}^{*}} ; 0\right)$ are given according to different cases:

(i) In the case where $P \in\left[0, \frac{\beta_{1}}{2 \theta \xi}\right]$, then $V_{p}(x)$ coincides with

$$
f(x)= \begin{cases}\beta_{1}\left(x-b_{p}^{*}\right)+\frac{\beta_{1}}{2 \theta \xi}, & x \geq b_{p}^{*}, \\ \frac{\beta_{1}}{2 \theta} \xi^{-\frac{1}{\xi+1}}\left(\frac{\xi+1}{\xi+a^{\pi_{p}^{*}}(0)} e^{2 \theta x}-1\right)^{\frac{\xi}{\xi+1}}, & 0 \leq x \leq b_{p}^{*},\end{cases}
$$

with $a^{\pi_{p}^{*}}(0)$ given by $(4.8)$ and $b_{p}^{*}=\frac{1}{2 \theta} \ln \left(1+\frac{a^{\pi_{p}^{*}}(0)}{\xi}\right)$. Correspondingly, $L_{p}^{\pi_{p}^{*}}$ is a barrier dividend strategy with level $b_{p}^{*}$ satisfying

$$
L_{t}^{\pi_{p}^{*}}=\left(x-b_{p}^{*}\right)^{+}+\int_{0}^{t} I_{\left\{X_{s}^{\pi_{p}^{*}}=b_{p}^{*}\right\}} \mathrm{d} L_{s}^{\pi_{p}^{*}} .
$$

The optimal ceded proportion $a^{\pi_{p}^{*}}$ is given by

$$
a^{\pi_{p}^{*}}(x)= \begin{cases}0, & x \geq b_{p}^{*}, \\ \left(\xi+a^{\pi_{p}^{*}}(0)\right) e^{-2 \theta x}-\xi, & 0 \leq x \leq b_{p}^{*},\end{cases}
$$


where $a^{\pi_{p}^{*}}(0) \in[0,1]$ is the unique root of the following equation

$$
\frac{\beta_{1}}{2 \theta} \xi^{-\frac{1}{\xi+1}}\left(\frac{\xi+1}{\xi+a^{\pi_{p}^{*}}(0)}-1\right)^{\frac{\xi}{\xi+1}}=P .
$$

(ii) In the case where $P \in\left[\frac{\beta_{1}}{2 \theta \xi}, \infty\right)$, then $V_{p}(x)$ equals to

$$
f(x)=\beta_{1} x+P .
$$

The associated optimal strategy is to distribute all surplus as dividends (by letting $b_{p}^{*}=0$ in (4.6)) and declare bankruptcy at once (by letting $0 \leq a^{\pi_{p}^{*}}(0)<1$ ).

Proof. (i) Note that $V_{p}(x)$ and $V_{r}(x)$ satisfy the same HJB equation but with different boundary conditions.

With the same argument, we can obtain the expressions of $f(x), a_{p}^{\pi_{p}^{*}}(x)$ and $L_{t}^{\pi_{p}^{*}}$. The boundary condition $f(0)=P$ (which is the same as equation (4.8)) gives the value of $a^{\pi_{p}^{*}}(0) \in\left[\tilde{a}_{0}, 1\right]$. In the case where $P \in\left[0, \frac{\beta_{1}}{2 \theta \xi}\right]$, we know that $a^{\pi_{p}^{*}}(0)$ exists. Obviously, it is not difficult to verify that $f(x)$ is indeed a twice continuously differentiable, increasing and concave solution of (4.3) and (4.4), and the optimality of $\pi_{p}^{*}$ can also be established as that in Appendix B. All conditions in Theorem 4.1 are satisfied.

(ii) In the case where $P \in\left[\frac{\beta_{1}}{2 \theta \xi}, \infty\right)$, (4.8) has no solution for $a^{\pi_{p}^{*}}(0)$. We conclude that $f(x)=\beta_{1} x+P, x \geq 0$ solves (4.3) and (4.4) now, because

$$
\begin{aligned}
\max _{0 \leq a \leq 1}\left\{\mathscr{A}^{a} f(x)\right\} & =\max _{0 \leq a \leq 1}\left\{\left(1-a^{2}\right) \theta \lambda \mu_{2}^{2} \beta_{1}-\delta\left(\beta_{1} x+P\right)\right\} \\
& =\theta \lambda \mu_{2}^{2} \beta_{1}-\delta\left(\beta_{1} x+P\right) \\
& =-\delta\left(P-\frac{\beta_{1}}{2 \theta \xi}\right)-\delta \beta_{1} x \leq 0
\end{aligned}
$$

and $f^{\prime}(x) \equiv \beta_{1}$ on $[0, \infty)$. Furthermore, we define a control strategy $\pi_{p}^{*}$ by letting the $b_{p}^{*}=0$ in (4.6) and $0 \leq a^{\pi_{p}^{*}}(0)<1$, then $f(x)=V\left(x, \pi_{p}^{*}\right)$ follows. The statement of $(i i)$ is true according to Theorem 4.1.

Remark 4.1. It is interesting to see $V_{p}(x)=f(x)$ behaves differently depending on the relationship between $P$ and $\frac{\beta_{1}}{2 \theta \xi}$. In fact, $\frac{\beta_{1}}{2 \theta \xi}=\beta_{1} \lambda \theta \mu_{2}^{2} / \delta$ is the present value of a perpetuity with discount rate $\delta$ and rate of income $\beta_{1} \lambda \theta \mu_{2}^{2}$, which is the expected rate of profit under the strategy of full retention. Therefore, when salvage value is greater than the present value of this perpetuity, then it is optimal for the insurer to declare bankruptcy and claim the salvage value. This strategy is called take-the-money-and-run by Loeffen and Renaud (2010, Theorem 1.1) and Liang and Young (2012, Remark 3.1).

\subsection{Restricted dividends}

In this subsection, we consider the optimal classical dividend problem where a ceiling $M>0$ is imposed on the dividend rate. Let $\bar{\pi}_{p}=\left(a^{\bar{\pi}_{p}} ; L^{\bar{\pi}_{p}} ; 0\right) \in \bar{\Pi}$ stand for the control process in which capital injection is not allowed. The performance function associated with $\bar{\pi}_{p}$ is defined by

$$
V\left(x, \bar{\pi}_{p}\right)=\mathrm{E}^{x}\left(\beta_{1} \int_{0}^{\tau^{\bar{\pi}_{p}}} e^{-\delta s} l_{s}^{\bar{\pi}_{p}} \mathrm{~d} s+P e^{-\delta \tau^{\bar{\pi}_{p}}}\right) .
$$

We focus on finding the value function

$$
\bar{V}_{p}(x)=\max _{\bar{\pi}_{p} \in \bar{\Pi}} V\left(x, \bar{\pi}_{p}\right)
$$

and the optimal strategy $\bar{\pi}_{p}^{*}=\left(a^{\bar{\pi}_{p}^{*}} ; L^{\bar{\pi}_{p}^{*}} ; 0\right) \in \bar{\Pi}$ such that $\bar{V}_{p}(x)=V\left(x, \bar{\pi}_{p}^{*}\right)$. From the stochastic control theory, $\bar{V}_{p}(x)$ should satisfy the following HJB equation

$$
\max _{0 \leq \bar{a} \leq 1,0 \leq l \leq M}\left\{\mathscr{A}^{\bar{a}} \bar{V}_{p}(x)+l\left(\beta_{1}-\bar{V}_{p}^{\prime}(x)\right)\right\}=0
$$

and the boundary condition

$$
\bar{V}_{p}(0)=P .
$$


Theorem 4.3. Let $h(x)$ be a twice continuously differentiable, increasing and concave solution of (4.13) and (4.14), then we have the following statements:

(i) For each $\bar{\pi}_{p} \in \bar{\Pi}$, we have $h(x) \geq V\left(x, \bar{\pi}_{p}\right)$. So $h(x) \geq \bar{V}_{p}(x)$ for all $x \geq 0$.

(ii) If there exists some strategy $\bar{\pi}_{p}^{*}=\left(a^{\bar{\pi}_{p}^{*}} ; L^{\bar{\pi}_{p}^{*}} ; 0\right)$ such that $h(x)=V\left(x, \bar{\pi}_{p}^{*}\right)$, then $h(x)=\bar{V}_{p}(x)$ and $\bar{\pi}_{p}^{*}$ is optimal.

Proof. The proof of $(i)$ is similar to Appendix A, it is omitted here. The result of $(i i)$ is obvious.

Theorem 4.4. The value function $\bar{V}_{p}(x)$ and the associated optimal strategy $\bar{\pi}_{p}^{*}=\left(a^{\bar{\pi}_{p}^{*}} ; L^{\bar{\pi}_{p}^{*}} ; 0\right)$ are given according to different cases:

(i) In the case where $P \in\left[0, \frac{\beta_{1}\left(1-\bar{a}^{*}\right)}{2 \theta \xi}\right]$, then $\bar{V}_{p}(x)$ coincides with

$$
h(x)=\left\{\begin{array}{lc}
\frac{\beta_{1} M}{\delta}+\frac{\beta_{1}}{\gamma} e^{\gamma\left(x-\bar{b}_{p}^{*}\right)}, & x>\bar{b}_{p}^{*}, \\
\frac{A}{2 \theta \xi}\left(\frac{\xi+1}{\xi+a^{\bar{\pi}_{p}^{*}}(0)} e^{2 \theta x}-1\right)^{\frac{\xi}{\xi+1}}, & 0 \leq x \leq \bar{b}_{p}^{*},
\end{array}\right.
$$

where $A=\beta_{1}\left(\xi+\bar{a}^{*}\right)^{\frac{\xi}{\xi+1}}\left(1-\bar{a}^{*}\right)^{\frac{1}{\xi+1}}, \bar{b}_{p}^{*}=\frac{1}{2 \theta} \ln \left(\frac{\xi+a_{p}^{*}(0)}{\xi+\bar{a}^{*}}\right)$ and $a^{\bar{\pi}_{p}^{*}}(0), \bar{a}^{*}, \gamma$ are roots of (4.18), (4.19) and (4.25), respectively. The optimal dividend strategy $L^{\bar{\pi}_{p}^{*}}$ is defined by the following dividend rate

$$
l^{\bar{\pi}_{p}^{*}}=\bar{l}_{p}^{*}(x)=\left\{\begin{array}{l}
M, \quad x>\bar{b}_{p}^{*} \\
0, \quad 0 \leq x \leq \bar{b}_{p}^{*}
\end{array}\right.
$$

The optimal ceded proportion $a^{\bar{\pi}_{p}^{*}}$ is given by

$$
a^{\bar{\pi}_{p}^{*}}(x)= \begin{cases}\bar{a}^{*}, & x>\bar{b}_{p}^{*}, \\ \left(\xi+a^{\bar{\pi}_{p}^{*}}(0)\right) e^{-2 \theta x}-\xi, & 0<x \leq \bar{b}_{p}^{*} .\end{cases}
$$

Here $a^{\bar{\pi}_{p}^{*}}(0) \in(0,1)$ is the unique solution of the following equation

$$
\frac{A}{2 \theta \xi}\left(\frac{\xi+1}{\xi+a^{\bar{\pi}_{p}^{*}}(0)}-1\right)^{\frac{\xi}{\xi+1}}=P
$$

$\bar{a}^{*} \in(0,1)$ is the positive root of the equation

$$
a^{2}+\left(\xi-1+\frac{2 M \theta \xi}{\delta}\right) a-\xi=0
$$

(ii) In the case where $P \in\left[\frac{\beta_{1}\left(1-\bar{a}^{*}\right)}{2 \theta \xi}, \infty\right)$, then

$$
h(x)=\frac{\beta_{1} M}{\delta}+\left(P-\frac{\beta_{1} M}{\delta}\right) e^{\gamma x} .
$$

The associated optimal strategy $L^{\bar{\pi}_{p}^{*}}$ and $a^{\bar{\pi}_{p}^{*}}$ can also be described by (4.16) and (4.17) but with $\bar{b}_{p}^{*}=0$.

Proof. (i) The value functions $\bar{V}_{p}(x)$ and $V_{p}(x)$ satisfy the same HJB equation but with different boundary conditions at $x=0$. So (4.15)-(4.17) can be obtained similarly. The value of $a^{\bar{\pi}_{p}^{*}}(0)$ is determined by $h(0)=P$, which is the same as equation (4.18). Note that $a_{p}^{\bar{\pi}_{p}^{*}}(0)$ must be larger than the fixed number $\bar{a}^{*}$, the value of the left hand side of (4.18) varies in the region $\left[0, \frac{\beta_{1}\left(1-\bar{a}^{*}\right)}{2 \theta \xi}\right]$, the solution for $a^{\bar{\pi}_{p}^{*}}(0)$ exists in this case. Finally, we can establish that $h(x)$ is indeed a twice continuously differentiable, increasing and concave solution of (4.13) and (4.14), and $h(x)=\bar{V}_{p}(x)=V\left(x, \bar{\pi}_{p}^{*}\right)$, the verification process is omitted.

(ii) Let us consider the opposite case with $P \in\left[\frac{\beta_{1}\left(1-\bar{a}^{*}\right)}{2 \theta \xi}, \infty\right)$. We conjecture that $h^{\prime}(x) \leq \beta_{1}$ for all $x \geq 0$. Then

$$
\max _{0 \leq \bar{a} \leq 1,0 \leq l \leq M}\left\{\mathscr{A}^{\bar{a}} h(x)+l\left(\beta_{1}-h^{\prime}(x)\right)\right\}=\max _{0 \leq \bar{a} \leq 1}\left\{\mathscr{A}^{\bar{a}} h(x)+M\left(\beta_{1}-h^{\prime}(x)\right)\right\}
$$

specifically,

$$
\max _{0 \leq \bar{a} \leq 1}\left\{\frac{1}{2}(1-\bar{a})^{2} \lambda \mu_{2}^{2} h^{\prime \prime}(x)+\left(\left(1-\bar{a}^{2}\right) \theta \lambda \mu_{2}^{2}-M\right) h^{\prime}(x)-\delta h(x)+\beta_{1} M\right\}=0 .
$$


Taking derivative with respect to $\bar{a}$ on the left hand side of (4.22) yields

$$
\frac{h^{\prime \prime}(x)}{h^{\prime}(x)}=-\frac{2 \theta a^{\bar{\pi}_{p}^{*}}(x)}{1-a^{\overline{\bar{\pi}}_{p}^{*}}(x)}
$$

where $a^{\bar{\pi}_{p}^{*}}(x)$ is the maximizer in (4.22). However, we guess that $a^{\bar{\pi}_{p}^{*}}(x) \equiv \bar{a}^{*}$ is a constant, then the value function takes the form

$$
h(x)=C+D e^{\gamma x}
$$

with

$$
\gamma=-\frac{2 \theta \bar{a}^{*}}{1-\bar{a}^{*}}
$$

By plugging (4.24) into (4.22), we deduce that $C=\frac{\beta_{1} M}{\delta}$, and $0<\bar{a}^{*}<1$ is given by (4.19). Then $D=P-\frac{\beta_{1} M}{\delta}$ can also be obtained by the boundary condition $h(0)=P$. We still need to verify the condition $h^{\prime}(x) \leq \beta_{1}$ for all $x \geq 0$, equivalently,

$$
h^{\prime}(0)=D \gamma \leq \beta_{1} \Leftrightarrow P \geq \frac{\beta_{1}}{\gamma}+\frac{\beta_{1} M}{\delta}=-\frac{\beta_{1}\left(1-\bar{a}^{*}\right)}{2 \theta \bar{a}^{*}}+\frac{\beta_{1} M}{\delta}=\frac{\beta_{1}\left(1-\bar{a}^{*}\right)}{2 \theta \xi}
$$

where the last step follows from (4.19). It is easy to verify that $h(x)$ is indeed a twice continuously differentiable, increasing and concave solution of (4.13) and (4.14), the optimality of $\bar{\pi}_{p}^{*}$ can be established by the same way as that in Appendix B.

Remark 4.2. All results obtained in Subsections 3.2 and 4.2 are compatible with those in Subsection 3.1 and Subsection 4.1, respectively, when the dividend ceiling $M$ goes to infinity. That is, the optimal control problem without dividend restrictions can be seen as the limiting optimal control problem with bounded dividend rate.

\section{The solution to the general optimal problems}

Now, based on the analysis in sections above, we can address the problem of maximizing the performance function over all admissible strategies. The two general optimal control problems raised in Section 2 can be solved completely.

\subsection{Unrestricted dividends}

In this subsection, we would deal with Problem 2.1 in general case. According to the stochastic control theory, $V(x)$ should satisfy the following HJB equation

$$
\max \left\{\max _{0 \leq a \leq 1} \mathscr{A}^{a} V(x), \beta_{1}-V^{\prime}(x), \mathscr{M} V(x)-V(x)\right\}=0,
$$

with boundary condition

$$
\max \{\mathscr{M} V(0)-V(0), P-V(0)\}=0 .
$$

Theorem 5.1. Let $v(x)$ be a twice continuously differentiable, increasing and concave solution of equations (5.1) and (5.2), then we have the following statements:

(i) For each $\pi \in \Pi$ we have $v(x) \geq V(x, \pi)$. So $v(x) \geq V(x)$ for all $x \geq 0$.

(ii) If there exists some strategy $\pi^{*}=\left(a^{\pi^{*}} ; L^{\pi^{*}} ; G^{\pi^{*}}\right)$ such that $v(x)=V\left(x, \pi^{*}\right)$, then $v(x)=V(x)$ and $\pi^{*}$ is optimal.

Proof. See the proof process in Appendix A.

Next, let us give two lemmas, which show the relationships between parameters and signs of some important quantities. For convenience, denote

$$
\hat{P}:=\frac{\beta_{1}}{2 \theta} \xi^{-\frac{1}{\xi+1}}\left(\frac{\xi+1}{\xi+\hat{a}_{0}}-1\right)^{\frac{\xi}{\xi+1}}
$$


which proves to be a critical level later. By the way, $\hat{P}$ takes value on $\left[0, \frac{\beta_{1}}{2 \theta \xi}\right]$ since $\hat{a}_{0} \in[0,1]$.

Lemma 5.1. The signs of $\mathscr{M} g(0)-g(0)$ and $P-g(0)$ can be determined as follows:

(i) In the case where $0<K \leq I(1), P \leq \hat{P}$ and $a^{\pi_{r}^{*}}(0) \leq a^{\pi_{p}^{*}}(0)$, it follows that $\mathscr{M} g(0)-g(0)=0$ and $P-g(0) \leq 0$.

(ii) In the case where $0<K \leq I(1), P \leq \hat{P}$ and $a^{\pi_{r}^{*}}(0)>a^{\pi_{p}^{*}}(0)$, it follows that $\mathscr{M} g(0)-g(0)=0$ and $P-g(0)>0$.

(iii) In the case where $0<K \leq I(1), P>\hat{P}$, it follows that $\mathscr{M} g(0)-g(0)=0$ and $P-g(0)>0$.

(iv) In the case where $K>I(1)$, it follows that $\mathscr{M} g(0)-g(0)<0$.

Proof. $(i)$ Theorem 3.2 shows that $\mathscr{M} g(0)-g(0)=0$ holds with some $a_{r}^{\pi_{r}^{*}}(0) \in\left(\hat{a}_{0}, 1\right]$ when $0<K \leq I(1)$. Furthermore, by observing the structures of (4.8) and (5.3), we know that the condition $P \leq \hat{P}$ leads to $a^{\pi_{p}^{*}}(0) \geq \hat{a}_{0}$, which makes the inequality $a_{r}^{\pi_{r}^{*}}(0) \leq a^{\pi_{p}^{*}}(0)$ possible. So $P-g(0) \leq 0$ holds if $a^{\pi_{r}^{*}}(0) \leq a^{\pi_{p}^{*}}(0)$.

(ii) In this case, the equality $\mathscr{M} g(0)-g(0)=0$ still holds. Similar to the analysis in $(i)$, the condition $a^{\pi_{r}^{*}}(0)>a^{\pi_{p}^{*}}(0)$ leads to $P-g(0)>0$.

(iii) In this case, the equality $\mathscr{M} g(0)-g(0)=0$ still holds. In addition, for the case of $\hat{P}<P \leq \frac{\beta_{1}}{2 \theta \xi}$, it follows that $0 \leq a^{\pi_{p}^{*}}(0)<\hat{a}_{0}<a^{\pi_{r}^{*}}(0)$, which leads to $P-g(0)>0$. For the case of $P \geq \frac{\beta_{1}}{2 \theta \xi}$, we have $g(0)<g\left(b_{r}^{*}\right)=\frac{\beta_{1}}{2 \theta \xi} \leq P$ since the function $g(x)$ is increasing.

(iv) Obviously, (iv) is a direct result of Theorem 3.2.

Lemma 5.2. The property of $P-f(0)=0$ is known. Moreover, we can derive the sign of $\mathscr{M} f(0)-f(0)$ according to the following cases:

(i) In the case where $0<K \leq I(1), P \leq \hat{P}$ and $a^{\pi_{p}^{*}}(0) \geq a^{\pi_{r}^{*}}(0)$, it follows that $\mathscr{M} f(0)-f(0) \geq 0$.

(ii) In the case where $0<K \leq I(1), P \leq \hat{P}$ and $a^{\pi_{p}^{*}}(0)<a^{\pi_{r}^{*}}(0)$, it follows that $\mathscr{M} f(0)-f(0)<0$.

(iii) In the case where $0<K \leq I(1), P>\hat{P}$, it follows that $\mathscr{M} f(0)-f(0)<0$.

(iv) In the case where $K>I(1)$, it follows that $\mathscr{M} f(0)-f(0)<0$.

Proof. (i) Observing the structures of (4.8) and (5.3), the condition $P \leq \hat{P}$ implies that $a^{\pi_{p}^{*}}(0) \geq \hat{a}_{0}$. Lemma 3.1 suggests that there exists some number $\zeta<b_{p}^{*}$ with $f^{\prime}(\zeta)=\Phi_{a_{p}^{\pi_{p}^{*}(0)}}(\zeta)=\beta_{2}$. Then, it follows that $f^{\prime}(x)-\beta_{2} \geq 0$ on $(0, \zeta]$ and $f^{\prime}(x)-\beta_{2}<0$ on $(\zeta, \infty)$ since $f(x)$ is concave. Consequently,

$$
\begin{aligned}
\mathscr{M} f(0)-f(0) & =\max _{y \geq 0}\left\{f(y)-\beta_{2} y-K-f(0)\right\} \\
& =\max _{y \geq 0}\left\{\int_{0}^{y}\left(f^{\prime}(x)-\beta_{2}\right) \mathrm{d} x\right\}-K \\
& =\int_{0}^{\zeta}\left(f^{\prime}(x)-\beta_{2}\right) \mathrm{d} x-K \\
& =I\left(a^{\pi_{p}^{*}}(0)\right)-K \\
& \geq I\left(a^{\pi_{r}^{*}}(0)\right)-K=0 .
\end{aligned}
$$

The above inequality follows from $a^{\pi_{p}^{*}}(0) \geq a^{\pi_{r}^{*}}(0)$.

(ii) Similar to the analysis in $(i)$, the condition $a^{\pi_{p}^{*}}(0)<a^{\pi_{r}^{*}}(0)$ leads to

$$
\mathscr{M} f(0)-f(0)=I\left(a^{\pi_{p}^{*}}(0)\right)-K<I\left(a^{\pi_{r}^{*}}(0)\right)-K=0 .
$$

(iii) In the case of $P>\hat{P}$, the number $a^{\pi_{p}^{*}}(0) \in\left[\hat{a}_{0}, 1\right]$ does not exist, we can not find the number $\zeta>0$ such that $f^{\prime}(\zeta)=\Phi_{a_{p}^{\pi_{p}^{*}(0)}}(\zeta)=\beta_{2}$. It implies that $f^{\prime}(x)<\beta_{2}$ holds for all $x \geq 0$ since $f(x)$ is concave. Thereby,

$$
\begin{aligned}
\mathscr{M} f(0)-f(0) & =\max _{y \geq 0}\left\{f(y)-\beta_{2} y-K-f(0)\right\} \\
& =\max _{y \geq 0}\left\{\int_{0}^{y}\left(f^{\prime}(x)-\beta_{2}\right) \mathrm{d} x\right\}-K<0 .
\end{aligned}
$$

(iv) From the above analysis we know the value of $\mathscr{M} f(0)-f(0)$ can not exceed $I(1)-K$, no matter the number $\zeta>0$ with $f^{\prime}(\zeta)=\beta_{2}$ exists or not. So $\mathscr{M} f(0)-f(0)<0$ follows.

Consequently, by comparing two different suboptimal models in Subsection 3.1 and Subsection 4.1, we 
can address the problem of maximizing the performance function $V(x, \pi)$ over all admissible strategies.

Theorem 5.2. Let $g(x)$ be the solution appearing in Theorem 3.2, and let $f(x)$ be the solution appearing in Theorem 4.2. Then we can solve Problem 2.1 as follows:

Case 1. In the case where $\mathscr{M} g(0)-g(0)=0$ and $P-g(0) \leq 0$, or equivalently, the following condition holds, (i) $0<K \leq I(1), P \leq \hat{P}$ and $a^{\pi_{r}^{*}}(0) \leq a^{\pi_{p}^{*}}(0)$,

then $V(x)=V_{r}(x)=\bar{g}(x)$ and $\pi^{*}$ coincides with $\pi_{r}^{*}=\left(a^{\pi_{r}^{*}} ; L^{\pi_{r}^{*}} ; G^{\pi_{r}^{*}}\right)$ appearing in Theorem 3.2.

Case 2. In the case where $\mathscr{M} f(0)-f(0) \leq 0$ and $P-f(0)=0$, or equivalently, one of the following conditions holds,

(ii) $0<K \leq I(1), P \leq \hat{P}$ and $a^{\pi_{r}^{*}}(0)>a^{\pi_{p}^{*}}(0)$;

(iii) $0<K \leq I(1)$ and $P>\hat{P}$;

(iv) $K \geq I(1)$

then $V(x)=V_{p}(x)=f(x)$ and $\pi^{*}$ coincides with $\pi_{p}^{*}=\left(a^{\pi_{p}^{*}} ; L^{\pi_{p}^{*}} ; G^{\pi_{p}^{*}}\right)$ appearing in Theorem 4.2.

Proof. See the proof process in Appendix B.

\subsection{Restricted dividends}

In this subsection, we would solve Problem 2.2 in general case. According to the stochastic control theory, $\bar{V}(x)$ should satisfy the following HJB equation

$$
\max \left\{\max _{0 \leq \bar{a} \leq 1,0 \leq l \leq M}\left\{\mathscr{A}^{\bar{a}} \bar{V}(x)+l\left(\beta_{1}-\bar{V}^{\prime}(x)\right)\right\}, \mathscr{M} \bar{V}(x)-\bar{V}(x)\right\}=0
$$

with boundary condition

$$
\max \{\mathscr{M} \bar{V}(0)-\bar{V}(0), P-\bar{V}(0)\}=0 .
$$

Theorem 5.3. Let $u(x)$ be a twice continuously differentiable, increasing and concave solution of equations (5.7) and (5.8), then we have the following statements:

(i) For each $\bar{\pi} \in \bar{\Pi}$, it follows that $u(x) \geq V(x, \bar{\pi})$. So $u(x) \geq \bar{V}(x)$ for all $x \geq 0$.

(ii) If there exists some strategy $\bar{\pi}^{*}=\left(a^{\bar{\pi}^{*}} ; L^{\bar{\pi}^{*}} ; G^{\bar{\pi}^{*}}\right)$ such that $u(x)=V\left(x, \bar{\pi}^{*}\right)$, then $u(x)=\bar{V}(x)$ and $\bar{\pi}^{*}$ is optimal.

Proof. Repeating a similar proof procedure as that in Appendix A, the results can be proved.

Similar to the discussion in Subsection 5.1, let us give following two lemmas, but the proofs are omitted. To facilitate the expression, we denote that

$$
\tilde{P}:=\frac{A}{2 \theta \xi}\left(\frac{\xi+1}{\xi+\tilde{a}_{0}}-1\right)^{\frac{\xi}{\xi+1}}
$$

which is also a critical level. By the way, $\tilde{P}$ takes value on $\left[0, \frac{\beta_{1}\left(1-\bar{a}^{*}\right)}{2 \theta \xi}\right]$ since $\tilde{a}_{0} \in[0,1]$.

Lemma 5.3. The signs of $\mathscr{M} w(0)-w(0)$ and $P-w(0)$ can be determined as follows.

(i) In the case where $0<K \leq \bar{I}(1), P \leq \tilde{P}$ and $a^{\bar{\pi}_{r}^{*}}(0) \leq a^{\bar{\pi}_{p}^{*}}(0)$, it has $\mathscr{M} w(0)-w(0)=0$ and $P-w(0) \leq 0$.

(ii) In the case where $0<K \leq \bar{I}(1), P \leq \tilde{P}$ and $a^{\bar{\pi}_{r}^{*}}(0)>a^{\bar{\pi}_{p}^{*}}(0)$, it has $\mathscr{M} w(0)-w(0)=0$ and $P-w(0)>0$.

(iii) In the case where $0<K \leq \bar{I}(1), P>\tilde{P}$, it has $\mathscr{M} w(0)-w(0)=0$ and $P-w(0)>0$.

(iv) In the case where $K>\bar{I}(1)$, it has $\mathscr{M} w(0)-w(0)<0$.

Lemma 5.4. The signs of $\mathscr{M} h(0)-h(0)$ and $P-h(0)$ can be determined as follows.

(i) In the case where $0<K \leq \bar{I}(1), P \leq \tilde{P}$ and $a^{\bar{\pi}_{p}^{*}}(0) \geq a^{\bar{\pi}_{r}^{*}}(0)$, it has $\mathscr{M} h(0)-h(0) \geq 0$ and $P-h(0)=0$.

(ii) In the case where $0<K \leq \bar{I}(1), P \leq \tilde{P}$ and $a^{\bar{\pi}_{p}^{*}}(0)<a^{\bar{\pi}_{r}^{*}}(0)$, it has $\mathscr{M} h(0)-h(0)<0$ and $P-h(0)=0$.

(iii) In the case where $0<K \leq \bar{I}(1), P>\tilde{P}$, it has $\mathscr{M} h(0)-h(0)<0$ and $P-h(0)=0$.

(iv) In the case where $K>\bar{I}(1)$, it has $\mathscr{M} h(0)-h(0)<0$ and $P-h(0)=0$.

Next, by comparing results in Subsection 3.2 and Subsection 4.2, we will identify Problem 2.2 of maximizing the performance function $V(x, \bar{\pi})$ over all admissible strategies. 
Theorem 5.4. Let $w(x)$ be the solution appearing in Theorem 3.4, and let $h(x)$ be the solution appearing in Theorem 4.4. Then the Problem 2.2 can be solved according to different cases:

Case 1. In the case where $\mathscr{M} w(0)-w(0)=0$ and $P-w(0) \leq 0$, i.e., the following condition holds,

(i) $0<K \leq \bar{I}(1), P \leq \tilde{P}$ and $a^{\bar{\pi}_{r}^{*}}(0) \leq a^{\bar{\pi}_{p}^{*}}(0)$,

then $\bar{V}(x)=\bar{V}_{r}(x)=w(x)$ and $\bar{\pi}^{*}$ coincides with $\bar{\pi}_{r}^{*}=\left(a^{\bar{\pi}_{r}^{*}} ; L^{\bar{\pi}_{r}^{*}} ; G^{\bar{\pi}_{r}^{*}}\right)$ appearing in Theorem 3.4.

Case 2. In the case where $\mathscr{M} h(0)-h(0)<0$ and $P-h(0)=0$, i.e., one of the following conditions holds,

(ii) $0<K \leq \bar{I}(1), P \leq \tilde{P}$ and $a^{\bar{\pi}_{p}^{*}}(0)<a^{\bar{\pi}_{r}^{*}}(0)$;

(iii) $0<K \leq \bar{I}(1), P>\tilde{P}$;

(iv) $K>\bar{I}(1)$,

then $\bar{V}(x)=\bar{V}_{p}(x)=h(x)$ and $\bar{\pi}^{*}$ coincides with $\bar{\pi}_{p}^{*}=\left(a^{\bar{\pi}_{p}^{*}} ; L^{\bar{\pi}_{p}^{*}} ; G^{\bar{\pi}_{p}^{*}}\right)$ appearing in Theorem 4.4.

Proof. Repeating a similar proof procedure as that in Appendix B, the results can be verified.

Remark 5.1. Zhou and Yuen (2012) have explored the optimal dividend, capital injection and reinsurance problem in the special case with $P=K=0$. All results there can be viewed as limiting forms of ours as $P \rightarrow 0$ and $K \rightarrow 0$. Due to the existence of the fixed cost and the salvage value, to inject capital is not always optimal when $K>0$ or $P>0$ in our model. The decision to inject new capitals or to declare bankruptcy depends on the costs of injections and the profitability in future.

\section{Appendix}

\section{A. Proofs of Theorem 5.1}

For each given strategy $\pi=\left(a^{\pi} ; L^{\pi} ; G^{\pi}\right) \in \Pi$, define $\Lambda_{L}^{\pi}=\left\{s: L_{s-}^{\pi} \neq L_{s}^{\pi}\right\}, \Lambda_{G}^{\pi}=\left\{s: G_{s-}^{\pi} \neq G_{s}^{\pi}\right\}=$ $\left\{\tau_{1}^{\pi}, \tau_{2}^{\pi}, \cdots, \tau_{n}^{\pi}, \cdots\right\}$. Let $\hat{L}_{t}^{\pi}=\sum_{s \in \Lambda_{L}^{\pi}, s \leq t}\left(L_{s}^{\pi}-L_{s-}^{\pi}\right)$ be the discontinuous part of $L_{t}^{\pi}$ and $\tilde{L}_{t}^{\pi}=L_{t}^{\pi}-\hat{L}_{t}^{\pi}$ be

the continuous part of $L_{t}^{\pi}$. Similarly, $\hat{G}_{t}^{\pi}$ and $\tilde{G}_{t}^{\pi}$ stand for the discontinuous and continuous parts of $G_{t}^{\pi}$ respectively. Then, applying the generalized Itô formula, we derive that

$$
\begin{aligned}
& e^{-\delta\left(t \wedge \tau^{\pi}\right)} v\left(X_{t \wedge \tau^{\pi}}^{\pi}\right)-v(x) \\
& =\int_{0}^{t \wedge \tau^{\pi}} e^{-\delta s} \mathscr{L}^{a^{\pi}} v\left(X_{s}^{\pi}\right) \mathrm{d} s+\int_{0}^{t \wedge \tau^{\pi}}\left(1-a^{\pi}\right) \sqrt{\lambda} \mu_{2} e^{-\delta s} v^{\prime}\left(X_{s}^{\pi}\right) \mathrm{d} B_{s}-\int_{0}^{t \wedge \tau^{\pi}} e^{-\delta s} v^{\prime}\left(X_{s}^{\pi}\right) \mathrm{d} \tilde{L}_{s}^{\pi} \\
& \quad+\int_{0}^{t \wedge \tau} e^{-\delta s} v^{\prime}\left(X_{s}^{\pi}\right) \mathrm{d} \tilde{G}_{s}^{\pi}+\sum_{s \in \Lambda_{G}^{\pi} \cup \Lambda_{L}^{\pi}, s \leq t \wedge \tau^{\pi}} e^{-\delta s}\left(v\left(X_{s}^{\pi}\right)-v\left(X_{s-}^{\pi}\right)\right) .
\end{aligned}
$$

The last term on the right hand side can be written as

$$
\begin{aligned}
& \sum_{s \in \Lambda_{G}^{\pi} \cup \Lambda_{L}^{\pi}, s \leq t \wedge \tau^{\pi}} e^{-\delta s}\left(v\left(X_{s}^{\pi}\right)-v\left(X_{s-}^{\pi}\right)\right) \\
= & \sum_{s \in \Lambda_{L}^{\pi}, s \leq t \wedge \tau^{\pi}} e^{-\delta s}\left(v\left(X_{s}^{\pi}\right)-v\left(X_{s-}^{\pi}\right)\right)+\sum_{s \in \Lambda_{G}^{\pi}, s \leq t \wedge \tau^{\pi}} e^{-\delta s}\left(v\left(X_{s}^{\pi}\right)-v\left(X_{s-}^{\pi}\right)\right) \\
\leq & -\sum_{s \in \Lambda_{L}^{\pi}, s \leq t \wedge \tau^{\pi}} e^{-\delta s} \beta_{1}\left(L_{s}^{\pi}-L_{s-}^{\pi}\right)+\sum_{n=1}^{\infty} e^{-\delta \tau_{n}^{\pi}}\left(\beta_{2} \xi_{n}^{\pi}+K\right) I_{\left\{\tau_{n}^{\pi} \leq t \wedge \tau^{\pi}\right\}}
\end{aligned}
$$

where the inequality is due to that $v(x)$ satisfies the HJB equation (5.1) with $v^{\prime}(x) \geq \beta_{1}$ and $\mathscr{M} v(x) \leq v(x)$. Moreover, in view of (5.1), the first term on the right hand side of (A.1) is non-positive. So substituting (A.2) into (A.1), we obtain

$$
\begin{aligned}
e^{-\delta\left(t \wedge \tau^{\pi}\right)} v\left(X_{t \wedge \tau^{\pi}}^{\pi}\right) \leq v(x) & +\int_{0}^{t \wedge \tau^{\pi}}\left(1-a^{\pi}\right) \sqrt{\lambda} \mu_{2} e^{-\delta s} v^{\prime}\left(X_{s}^{\pi}\right) \mathrm{d} B_{s}-\beta_{1} \int_{0}^{t \wedge \tau^{\pi}} e^{-\delta s} \mathrm{~d} L_{s}^{\pi} \\
& +\sum_{n=1}^{\infty} e^{-\delta \tau_{n}^{\pi}}\left(\beta_{2} \xi_{n}^{\pi}+K\right) I_{\left\{\tau_{n}^{\pi} \leq t \wedge \tau^{\pi}\right\}} .
\end{aligned}
$$


Since $v(x)$ is an increasing function and $v(0) \geq P$, we have

$$
\begin{aligned}
e^{-\delta\left(t \wedge \tau^{\pi}\right)} P \leq v(x) & +\int_{0}^{t \wedge \tau^{\pi}}\left(1-a^{\pi}\right) \sqrt{\lambda} \mu_{2} e^{-\delta s} v^{\prime}\left(X_{s}^{\pi}\right) \mathrm{d} B_{s}-\beta_{1} \int_{0}^{t \wedge \tau^{\pi}} e^{-\delta s} \mathrm{~d} L_{s}^{\pi} \\
& +\sum_{n=1}^{\infty} e^{-\delta \tau_{n}^{\pi}}\left(\beta_{2} \xi_{n}^{\pi}+K\right) I_{\left\{\tau_{n}^{\pi} \leq t \wedge \tau^{\pi}\right\}} .
\end{aligned}
$$

The stochastic integral with respect to the Brownian motion in (A.4) is a uniformly integrable martingale, if $v^{\prime}(x)$ is bounded. Taking expectation and limit on both sides of (A.4) yields

$$
v(x) \geq \mathrm{E}^{x}\left(\beta_{1} \int_{0}^{\tau^{\pi}} e^{-\delta s} \mathrm{~d} L_{s}^{\pi}-\sum_{n=1}^{\infty} e^{-\delta \tau_{n}^{\pi}}\left(\beta_{2} \eta_{n}^{\pi}+K\right) I_{\left\{\tau_{n}^{\pi} \leq \tau^{\pi}\right\}}+P e^{-\delta \tau^{\pi}}\right)=V(x, \pi) .
$$

Consequently, $v(x) \geq V(x)$ by (2.6). Applying the method in Cadenillas et al. (2006), we can also prove above results when $v^{\prime}(x)$ is unbounded by modifying above proof process, it is omitted here.

\section{B. Proofs of Theorem 5.2}

(1) If $P-g(0) \leq 0$ and $\mathscr{M} g(0)=g(0)$, then $g(x)$ satisfies conditions of Theorem 5.1, $g(x) \geq V(x)$. Since $\mathscr{L}^{a_{r}^{*}} g\left(X_{t}^{\pi_{r}^{*}}\right)=0$ for $0 \leq X_{t}^{\pi_{r}^{*}} \leq b_{r}^{*}$, one has

$$
\int_{0}^{t \wedge \tau^{\pi_{r}^{*}}} e^{-\delta s} \mathscr{L}^{a^{\pi_{r}^{*}}} g\left(X_{s}^{\pi_{r}^{*}}\right) \mathrm{d} s=\int_{0}^{t \wedge \tau^{\pi_{r}^{*}}} e^{-\delta s} \mathscr{L}^{a^{\pi_{r}^{*}}} g\left(X_{s}^{\pi_{r}^{*}}\right) I_{\left\{0 \leq X_{s}^{\pi_{r}^{*}} \leq b_{r}^{*}\right\}} \mathrm{d} s=0
$$

Furthermore, (3.5)-(3.7) and (3.9) indicate that

$$
\begin{aligned}
& \sum_{s \in \Lambda_{G}^{\pi_{r}^{*}} \cup \Lambda_{L}^{\pi_{r}^{*}}, s \leq t \wedge \tau^{\pi_{r}^{*}}} e^{-\delta s}\left(g\left(X_{s}^{\pi_{r}^{*}}\right)-g\left(X_{s-}^{\pi_{r}^{*}}\right)\right) \\
= & \sum_{s \in \Lambda_{L}^{\pi_{r}^{*}}, s \leq t \wedge \tau^{\pi_{r}^{*}}} e^{-\delta s}\left(g\left(X_{s}^{\pi_{r}^{*}}\right)-g\left(X_{s-}^{\pi_{r}^{*}}\right)\right) I_{\left\{X_{s}^{\pi_{r}^{*}}=b_{r}^{*}\right\}}+\sum_{s \in \Lambda_{G}^{\pi_{r}^{*}}, s \leq t \wedge \tau^{\pi_{r}^{*}}} e^{-\delta s}\left(g\left(X_{s}^{\pi_{r}^{*}}\right)-g\left(X_{s-}^{\pi_{r}^{*}}\right)\right) I_{\left\{X_{s-}^{\pi_{r}^{*}}=0\right\}} \\
= & -\sum_{s \in \Lambda_{L}^{\pi_{r}^{*}}, s \leq t \wedge \tau^{\pi_{r}^{*}}} e^{-\delta s} \beta_{1}\left(L_{s}^{\pi_{r}^{*}}-L_{s-}^{\pi_{r}^{*}}\right)+\sum_{n=1}^{\infty} e^{-\delta \tau_{n}^{\pi_{r}^{*}}}\left(\beta_{2} \xi_{n}^{\pi_{r}^{*}}+K\right) I_{\left\{\tau_{n}^{\pi_{r}^{*}} \leq t \wedge \tau^{\pi_{r}^{*}}\right\}} .
\end{aligned}
$$

Replacing $\pi, \tau^{\pi}, v$ by $\pi_{r}^{*}, \tau_{r}^{\pi_{r}^{*}}=\infty, g$ in Itô formula (A.1) and taking expectations, we have

$$
g(x)=\mathrm{E}^{x}\left[e^{-\delta t} g\left(X_{t}^{\pi_{r}^{*}}\right)\right]+\mathrm{E}^{x}\left(\beta_{1} \int_{0}^{t} e^{-\delta s} \mathrm{~d} L_{s}^{\pi_{r}^{*}}-\sum_{n=1}^{\infty} e^{-\delta \tau_{n}^{\pi_{r}^{*}}}\left(\beta_{2} \xi_{n}^{\pi_{r}^{*}}+K\right) I_{\left\{\tau_{n}^{\pi_{r}^{*}} \leq t\right\}}\right) .
$$

Letting $t \rightarrow \infty$, the first term on the right hand side vanishes, then we obtain

$$
g(x)=\mathrm{E}^{x}\left(\beta_{1} \int_{0}^{\infty} e^{-\delta s} \mathrm{~d} L_{s}^{\pi_{r}^{*}}-\sum_{n=1}^{\infty} e^{-\delta \tau_{n}^{\pi_{r}^{*}}}\left(\beta_{2} \xi_{n}^{\pi_{r}^{*}}+K\right) I_{\left\{\tau_{n}^{\pi_{r}^{*}}<\infty\right\}}\right)=V\left(x, \pi_{r}^{*}\right),
$$

which, together with $g(x) \geq V(x)$, establishes that $g(x)=V(x)=V\left(x, \pi_{r}^{*}\right)$, and $\pi_{r}^{*}$ is the associated optimal strategy.

(2) If $P-f(0)=0$ and $\mathscr{M} f(0) \leq f(0)$, then $f(x)$ satisfies conditions of Theorem 5.1, so $f(x) \geq V(x)$. Since $\mathscr{L}^{a_{p}^{\pi_{p}^{*}}} f\left(X_{t}^{\pi_{p}^{*}}\right)=0$ for $0 \leq X_{t}^{\pi_{p}^{*}} \leq b_{p}^{*}$, one has

$$
\int_{0}^{t \wedge \tau_{p}^{*}} e^{-\delta s} \mathscr{L}^{a^{\pi_{p}^{*}}} f\left(X_{s}^{\pi_{p}^{*}}\right) \mathrm{d} s=\int_{0}^{t \wedge \tau_{p}^{*}} e^{-\delta s} \mathscr{L}^{a^{\pi_{p}^{*}}} f\left(X_{s}^{\pi_{p}^{*}}\right) I_{\left\{0 \leq X_{s}^{\pi_{p}^{*}} \leq b_{p}^{*}\right\}} \mathrm{d} s=0 .
$$


Moreover, under the strategy $\pi_{p}^{*}=\left(a^{\pi_{p}^{*}} ; L^{\pi_{p}^{*}} ; 0\right) \in \Pi$, the capital injection never occurs, $\Lambda_{G}^{\pi_{p}^{*}}$ is a null set. Thereby, together with (4.6), it follows that

$$
\begin{aligned}
\sum_{\substack{\pi_{G}^{*} \\
\pi_{p}^{*} \cup \Lambda_{L}^{\pi_{p}^{*}}, s \leq t \wedge \tau_{p}^{\pi_{p}^{*}}}} e^{-\delta s}\left(f\left(X_{s}^{\pi_{p}^{*}}\right)-f\left(X_{s-}^{\pi_{p}^{*}}\right)\right) & =\sum_{s \in \Lambda_{L}^{\pi_{p}^{*}}, s \leq t \wedge \tau^{\pi_{p}^{*}}} e^{-\delta s}\left(f\left(X_{s}^{\pi_{p}^{*}}\right)-f\left(X_{s-}^{\pi_{p}^{*}}\right)\right) I_{\left\{X_{s}^{\pi_{p}^{*}}=b_{p}^{*}\right\}} \\
& =\sum_{s \in \Lambda_{L}^{\pi_{p}^{*}}, s \leq t \wedge \tau^{\pi_{p}^{*}}} \beta_{1} e^{-\delta s}\left(L_{s}^{\pi_{p}^{*}}-L_{s-}^{\pi_{p}^{*}}\right) .
\end{aligned}
$$

Replacing $\pi, \tau, v$ by $\pi_{p}^{*}, \tau_{p}^{\pi_{p}^{*}}, f$, respectively, in Itô formula (A.1) and taking expectations, we have

$$
f(x)=\mathrm{E}^{x}\left(\beta_{1} \int_{0}^{t \wedge \tau^{\pi_{p}^{*}}} e^{-\delta s} \mathrm{~d} L_{s}^{\pi_{p}^{*}}+f\left(X_{t \wedge \tau_{p}^{\pi_{p}^{*}}}^{\pi_{p}^{*}}\right) e^{-\delta\left(t \wedge \tau^{\pi_{p}^{*}}\right)}\right) .
$$

Letting $t \rightarrow \infty$ yields

$$
f(x)=\mathrm{E}^{x}\left(\beta_{1} \int_{0}^{\tau^{\pi_{p}^{*}}} e^{-\delta s} \mathrm{~d} L_{s}^{\pi_{p}^{*}}+P e^{-\delta \tau^{\pi_{p}^{*}}}\right)=V\left(x, \pi_{p}^{*}\right),
$$

which, together with $f(x) \geq V(x)$, confirms that $f(x)=V(x)=V\left(x, \pi_{p}^{*}\right)$, and $\pi_{p}^{*}$ is the associated optimal strategy.

\section{Acknowledgments}

This work was supported by the Research Grants Council of the Hong Kong Special Administrative Region, China (project No. HKU 706611P), National Natural Science Foundation of China(11101205,11231005, 71071071), Doctoral Program Foundation of the Ministry of Education of China (20110076110004), The Fundamental Research Funds for the Central Universities, A Project Funded by the Priority Academic Program Development of Jiangsu Higher Education Institutions.

\section{References}

[1] Asmussen, S. and Taksar, M., 1997. Controlled diffusion models for optimal dividend pay-out. Ins.: Mathematics Econ. 20(1), $1-15$.

[2] Avram, F., Palmowski, Z. and Pistorius, M. R., 2007. On the optimal dividend problem for a spectrally negative Lévy process. Ann. Appl. Probab. 17(1), 156-180.

[3] Azcue, P. and Muler, N., 2012. Optimal dividend policies for compound Poisson processes: The case of bounded dividend rates. Ins.: Mathematics Econ. 51(1), 26-42.

[4] Bai, L., Guo, J. and Zhang, H., 2010. Optimal excess-of-loss reinsurance and dividend payments with both transaction costs and taxes. Quant. Financ. 10(10), 1163-1172.

[5] Belhaj, M., 2010. Optimal dividend payments when cash reserves follow a jump-diffusion process. Math. Financ. 20(2), 313-325.

[6] Cadenillas, A., Choulli, T., Taksar, M. and Zhang, L., 2006. Classical and impulse stochastic control for the optimization of the dividend and risk policies of an insurance firm. Math. Financ. 16(1), 181-202.

[7] Choulli, T., Taksar, M. and Zhou, X.Y., 2003. A diffusion model for optimal dividend distribution for a company with constraints on risk control. SIAM J. Control. Optim. 41(6), 1946-1979.

[8] Dai, H., Liu, Z. and Luan, N., 2010. Optimal dividend strategies in a dual model with capital injections. Math. Method. Oper. Res. 72(1), 129-143.

[9] Gerber, H.U. and Shiu, E.S.W., 2006. On optimal dividends strategies in the compound poisson model. N. Amer. Actuarial J. 10(2), 76-93.

[10] Gerber, H.U., Lin, X. S. and Yang, H., 2006. A note on the dividends-penalty identity and the optimal dividend barrier. ASTIN Bull. 36(2), 489-503.

[11] He, L. and Liang, Z.X., 2009. Optimal financing and dividend control of the insurance company with fixed and proportional transaction costs. Ins.: Mathematics Econ. 42(3), 88-94.

[12] H $\phi$ gaard, B. and Taksar, M., 2004. Optimal dynamic portfolio selection for a corporation with controllable risk and dividend distribution policy. Quant. Financ. 9(2), 153-182.

[13] Kulenko, N. and Schmidli, H., 2008. Optimal dividend strategies in a Cramér-Lundberg model with capital injections. Ins.: Mathematics Econ. 43(2), 270-278.

[14] Liang, Z. and Young, V.R., 2012. Dividends and reinsurance under a penalty for ruin. Ins.: Mathematics Econ. 50(3), $437-445$.

[15] Loeffen, R.L. and Renaud, J.F., 2010. De Finetti's optimal dividends problem with an affine penalty function at ruin. Ins.: Mathematics Econ. 46(1), 98-108.

[16] L $\phi \mathrm{kka}$, A. and Zervos, M., 2008. Optimal dividend and issuance of equity policies in the presence of proportional costs. Ins.: Mathematics Econ. 42(3), 954-961.

[17] Meng, H. and Siu, T., 2011. On optimal reinsurance, dividend and reinvestment strategies. Econ. Model. 28, 211-218.

[18] Paulsen, J., 2008. Optimal dividend payments and reinvestments of diffusion processes with both fixed and proportional costs. SIAM J. Control. Optim. 47(5), 2201-2226.

[19] Peng, X., Chen, M. and Guo, J., 2012. Optimal dividend and equity issuance problem with proportional and fixed transaction costs. Ins.: Mathematics Econ. 51(3), 576-585.

[20] Sethi, S.P. and Taksar, M., 2002. Optimal financing of a corporation subject to random returns. Math. Financ. 12, 155-172. 
[21] Taksar, M., 2000. Dependence of the optimal risk control decisions on the terminal value for a financial corporation. Ann. Oper. Res. 98(1), 89-99.

[22] Thonhauser, S. and Albrecher, H., 2007. Dividend maximization under consideration of the time value of ruin. Ins.: Mathematics Econ. 41(1), 163-184.

[23] Yao, D., Yang, H. and Wang, R., 2010. Optimal financing and dividend strategies in a dual model with proportional costs. J Ind. Manag. Optim. 6, 761-777.

[24] Yao, D., Yang, H. and Wang, R., 2011. Optimal dividend and capital injection problem in the dual model with proportional and fixed transaction costs. Eur. J. Oper. Res. 211(3), 568-576.

[25] Zhou, M. and Yuen, K.C., 2012. Optimal reinsurance and dividend for a diffusion model with capital injection: Variance premium principle. Econ. Model. 29, 198-207. 